\title{
Approximation to a Parabolic System Modeling the Thermoelastic Contacts of Two Rods
}

\author{
Jennifer J. Zhao \\ Department of Mathematics and Statistics \\ University of Michigan - Dearborn \\ Dearborn, Michigan 48128
}

Received November 19, 1996; accepted August 11, 1997

\begin{abstract}
In this article, we study a sequence of finite difference approximate solutions to a parabolic system, which models two dissimilar rods that may come into contact as a result of thermoelastic expansion. We construct the approximate solutions based on a set of finite difference schemes to the system, and we will prove that the approximate solutions converge strongly to the exact solutions. Moreover, we obtain and prove rigorously the error bound, which measures the difference between the exact solutions and approximate solutions in a reasonable norm. (C) 1998 John Wiley \& Sons, Inc. Numer Methods Partial Differential Eq 14: 1-25, 1998
\end{abstract}

Keywords: Finite difference; thermoelastic contact; nonlinear parabolic system

\section{INTRODUCTION}

The purpose of this article is to prove convergence and derive the error bound for a system of finite difference approximations to a parabolic system modeling the thermoelastic contacts of two dissimilar rods. Problems involving thermoelastic contact arise naturally in many applied situations, particularly those involving industrial processing, where two or more materials may come into contact or may lose contact as a result of thermoelastic expansion or contraction. The model of this article is one such situation: the mechanical behavior of two dissimilar rods, which are each fixed at one end but which may come into contact at their free ends as a result of thermoelastic expansions within finite time. Our model of the mechanical behavior originally consisted of a system of energy and elastic equations involving the temperature and displacement of each rod. The problem was then reformulated and reduced to solving an initial-boundary value problem for a nonlinear parabolic system containing only the two temperatures (see [1]).

The physical setting consists of two thin rods, each of which is clamped at one end but which may come into contact at their free ends. We assume that the process is independent of all but the horizontal variable. We use $\theta(x, t)$ to represent the temperature of the left rod on $0 \leq x \leq l_{1}$ and $\psi(x, t)$ to represent the temperature of the right rod on $l_{2} \leq x \leq 1, l_{1} \leq l_{2}$ both in nondimensional units. The fixed ends occur at $x=0$ and $x=1$, while the ends $x=l_{1}, x=l_{2}$ are free to expand 
or contact. We let $\tilde{g}=l_{2}-l_{1}$ to denote the initial gap between the rods in the reference configuration and the physical setting is displayed in Figure 1.

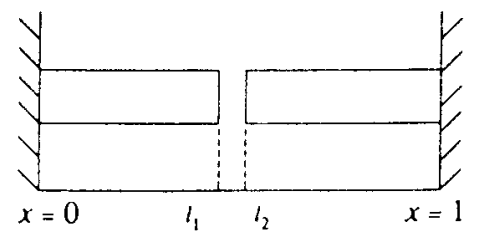

FIG. 1.

The complete parabolic system of equations is

$$
\begin{gathered}
\left(1+a_{1}^{2}\right) \theta_{t}-\theta_{x x}=\alpha_{1} g_{t}(\theta, \psi), 0 \leq x \leq l_{1}, t>0, \\
\left(d+a_{2}^{2}\right) \psi_{t}-\psi_{x x}=\alpha_{2} g_{t}(\theta, \psi), l_{2} \leq x \leq 1, t>0,
\end{gathered}
$$

where

$$
\begin{aligned}
g(\theta, \psi)(t) & =\max \left\{a_{1} \int_{0}^{l_{1}} \theta(x, t) d x+a_{2} \int_{l_{2}}^{1} \psi(x, t) d x-\tilde{g} ; 0\right\}, \\
\alpha_{1} & =\frac{a_{1} \lambda}{l_{1}(1+\lambda)}, \alpha_{2}=\frac{a_{2} \lambda}{\left(1-l_{2}\right)(1+\lambda)}, \tilde{g}=l_{2}-l_{1} .
\end{aligned}
$$

The initial condition is

$$
\theta(x, 0)=\tilde{\theta}(x), 0 \leq x \leq l_{1} ; \psi(x, 0)=\tilde{\psi}(x), l_{2} \leq x \leq 1,
$$

and boundary conditions are

$$
\begin{gathered}
\theta(0, t)=\psi(1, t)=0, t \geq 0, \\
-k_{1} \theta_{x}\left(l_{1}, t\right)=k\left(\theta\left(l_{1}, t\right)-\psi\left(l_{2}, t\right)\right), t \geq 0, \\
-k_{2} \psi_{x}\left(l_{2}, t\right)=k\left(\theta\left(l_{1}, t\right)-\psi\left(l_{2}, t\right)\right), t \geq 0 .
\end{gathered}
$$

$a_{1}, a_{2}$, and $d$ are positive constants related to material properties of the rods; $k_{1}, k_{2}$ are the heat condition coefficients and $k$ is the heat exchange coefficient; $\lambda$ is the elastic modulus. For most materials that arise in practice, $a_{1}$ and $a_{2}$ are very small, therefore, we will assume that $\alpha_{1}, \alpha_{2}$, which are defined in (1.4), are also small. The exact smallness conditions will be specified later. The current model is rather complicated in the sense that the two temperatures are coupled not only by a nonlinear system of equations, but also in the boundary conditions. The two source terms on the right hand side of (1.1), (1.2) are nonlocal as well as nonlinear. The system is also unusual in that each temperature function is defined on a different domain.

The particular model has been studied previously in [1] and [2]. In [1], they proved that under appropriate assumptions of the data, the system has a unique strong solution, i.e., a solution where each temperature lies in a Sobolev space. In [2], they presented some numerical simulations to the system, but there was no report of the convergence of those simulations and there was no error bound analysis either. Our goal in this article is, therefore, to construct a sequence of approximate (numerical) solutions to the system and to understand the convergence properties 
of those solutions in a rigorous mathematical way. More numerical works to related physical problems can be found in [2] and [3]. But in each case, the problem involves but a single temperature and a single displacement, so that decoupling leads to one nonlinear equation for a single temperature.

We construct the approximate solutions based on a set of finite difference schemes that are implicit, but linear. We show that the schemes can be solved easily for all time. We also establish a priori energy estimates to the approximate solutions; these are similar to those satisfied by the exact solutions, and are required to derive the weak truncation errors. We then show that the approximate solutions converge to the exact solutions in a stronger sense, i.e., stronger than the sense of distribution. Finally, we apply the weak truncation error estimates in obtaining the bound for the difference between the exact and the approximate solutions. The overall mesh requirement is essentially the standard stability requirement by a standard scheme to the parabolic equation.

We assume that $\tilde{\theta}(x), \tilde{\psi}(x)$ are smooth enough to satisfy the following conditions:

$$
\tilde{C}_{0}(x)=\|\tilde{\theta}\|_{H^{2}\left(0, l_{1}\right)}^{2}+\|\tilde{\psi}\|_{H^{2}\left(l_{2}, 1\right)}^{2}+\left(\tilde{\theta}\left(l_{1}\right)-\tilde{\psi}\left(l_{2}\right)\right)^{2}<\infty .
$$

We now construct the schemes. Let $\Delta t$ be the time increment, $\Delta x$ be the space increment such that $1=M \Delta x, l_{1}=M_{1} \Delta x, l_{2}=\left(M-M_{2}\right) \Delta x$, where $M, M_{1}, M_{2}$ are positive integers such that $M_{1} \leq M_{2} \leq M$. Throughout the article, we will use $\delta, \delta^{2}$ to represent the operators:

$$
\delta w_{j}=\frac{w_{j+1 / 2}-w_{j-1 / 2}}{\Delta x}, \delta w_{j+1 / 2}=\frac{w_{j+1}-w_{j}}{\Delta x}, \delta^{2} w_{j}=\frac{w_{j+1}-2 w_{j}+w_{j-1}}{\Delta x^{2}},
$$

and we often use the following summation by parts formulae:

$$
\begin{gathered}
\sum_{j=L_{1}}^{L_{2}} \delta w_{j} z_{j} \Delta x=-\sum_{j=L_{1}}^{L_{2}-1} w_{j+1 / 2} \delta z_{j+1 / 2} \Delta x+w_{L_{2}+1 / 2} z_{L_{1}}-w_{L_{1}-1 / 2} z_{L_{1}}, \\
\sum_{j=L_{1}}^{L_{2}} \delta w_{j+1 / 2} z_{j+1 / 2} \Delta x=-\sum_{j=L_{1}+1}^{L_{2}} w_{j} \delta z_{j} \Delta x+w_{L_{2}+1} z_{L_{2}+1 / 2}-w_{L_{1}+1 / 2} z_{L_{1}+1 / 2}
\end{gathered}
$$

where $L_{1}, L_{2}$ are integers. The two approximate temperatures $\left\{\theta_{j}^{q}\right\},\left\{\psi_{j}^{q}\right\}$ are then computed from the following implicit, but linear schemes:

$$
\begin{gathered}
\left(1+a_{1}^{2}\right) \frac{\theta_{j}^{q+1}-\theta_{j}^{q}}{\Delta t}-\delta^{2} \theta_{j}^{q+1}=\alpha_{1} \frac{g\left(\theta^{q}, \psi^{q}\right)-g\left(\theta^{q-1}, \psi^{q-1}\right)}{\Delta t}, 0 \leq j \leq M_{1}, \\
\left(d+a_{2}^{2}\right) \frac{\psi_{j}^{q+1}-\psi_{j}^{q}}{\Delta t}-\delta^{2} \psi_{j}^{q+1}=\alpha_{2} \frac{g\left(\theta^{q}, \psi^{q}\right)-g\left(\theta^{q-1}, \psi^{q-1}\right)}{\Delta t}, M_{2} \leq j \leq M, \\
g\left(\theta^{q}, \psi^{q}\right)=\max \left\{a_{1} \sum_{j=1}^{M_{1}} \theta_{j}^{q} \Delta x+a_{2} \sum_{j=M_{2}}^{M} \psi_{j}^{q} \Delta x-\tilde{g} ; 0\right\}, q \geq 0 .
\end{gathered}
$$

We take the following initial data

$$
\theta_{j}^{0}=\tilde{\theta}\left(x_{j}\right), 0 \leq j \leq M_{1} ; \psi_{j}^{0}=\tilde{\psi}\left(x_{j}\right), M_{2} \leq j \leq M,
$$

and the boundary conditions:

$$
\theta_{0}^{q}=0 ;-k_{1} \delta \theta_{M_{1}+1 / 2}^{q}=-k_{1} \frac{\theta_{M_{1}+1}^{q}-\theta_{M_{1}}^{q}}{\Delta x}=k\left(\theta_{M_{1}}^{q}-\psi_{M_{2}}^{q-1}\right),
$$




$$
\psi_{M}^{q}=0 ;-k_{2} \delta \psi_{M_{2}-1 / 2}^{q}=-k_{2} \frac{\psi_{M_{2}}^{q}-\psi_{M_{2}-1}^{q}}{\Delta x}=k\left(\theta_{M_{1}}^{q}-\psi_{M_{2}}^{q}\right) .
$$

In order to make the schemes complete, we also extend the schemes to

$$
\theta_{j}^{-1}=\theta_{j}^{0}, 0 \leq j \leq M_{1} ; \psi_{j}^{-1}=\psi_{j}^{0}, M_{2} \leq j \leq M,
$$

and

$$
\theta_{-1}^{q}=-\theta_{1}^{q}, \psi_{M+1}^{q}=-\psi_{M-1}^{q}, 0 \leq q \leq N
$$

We can prove that

$$
\begin{aligned}
C_{0}=\sum_{j=0}^{M_{1}}\left(\left(\theta_{j}^{0}\right)^{2}+\left(\delta^{2} \theta_{j}^{0}\right)^{2}\right) \Delta x & +\sum_{j=0}^{M_{1}-1}\left(\delta \theta_{j+1 / 2}^{0}\right)^{2} \Delta x+\sum_{j=M_{2}}^{M}\left(\left(\psi_{j}^{0}\right)^{2}+\left(\delta^{2} \psi_{j}^{0}\right)^{2}\right) \Delta x \\
& +\sum_{j=M_{2}}^{M-1}\left(\delta \psi_{j+1 / 2}^{0}\right)^{2} \Delta x+\left(\theta_{M_{1}}^{0}-\psi_{M_{2}}^{0}\right)^{2} \leq 5 \tilde{C}_{0}<\infty .
\end{aligned}
$$

Since the source terms are nonlocal as well as nonlinear, no standard results in the parabolic theory can be quoted here. Instead, we derive a priori energy estimates peculiar to this particular problem, and use the estimates to prove convergence and derive error bound. Comparable results can also be found in [4]-[8], where finite difference approximate solutions to other nonlinear parabolic system equations were studied. But those schemes are all implicit and nonlinear, which means a system of nonlinear equations must be solved at each time-step. Moreover, the initial data to those systems were required to be sufficiently small. By contrast, our schemes are implicit, but linear, which is quite easy to implement, and there is no smallness requirement to the initial data. It is also amenable to a complete rigorous convergence and error bound analysis.

The plan of the article is as follows. In Section II, we derive a priori energy estimates to the solutions $\left\{\theta_{j}^{q}\right\},\left\{\psi_{j}^{q}\right\}$ of the schemes, and prove that the schemes are solvable. Then, in Section III, we construct the approximate solutions $\left\{\theta^{h}\right\},\left\{\psi^{h}\right\}$, and we apply the estimates obtained in Section II to derive bounds for the weak truncation errors, which measure the extent to which the approximation solutions fail to be exact solutions. In Section IV, we prove that the approximate solutions converge and we apply the weak truncation error estimates in deriving the error bound. Finally, we provide some computational results in Section V.

Most of the estimates occuring in the following sections are long and technical; many of them are symmetric between $\theta$ and $\psi$. We have, therefore, omitted most of the repeating details.

\section{SOLVABILITY AND A PRIORI ENERGY ESTIMATES}

In this section, we will prove solvability and derive various energy estimates of the schemes.

Theorem 2.1. $\quad$ Assume that schemes (1.13)-(1.20) have been solved to $t^{n}\left(t^{n} \geq \Delta t\right)$, then the schemes can be advanced from $t^{n}$ to $t^{n+1}$ if $\Delta x$ is chosen to satisfy

$$
\Delta x \leq \min \left\{\frac{2 k_{1}}{k}, \frac{2 k_{2}}{k}\right\} .
$$

Proof. We will only prove the solvability for $\left\{\theta_{j}^{n}\right\}$; the proof for $\left\{\psi_{j}^{n}\right\}$ is similar and will be omitted. To advance $\left\{\theta_{j}^{n}\right\}$ to $\left\{\theta_{j}^{n+1}\right\}$, we need to solve the following system of linear equations:

$$
A \theta_{j}^{n+1}=B\left(\theta_{j}^{n}, \theta_{j}^{n-1}\right)
$$


where

$$
\theta_{j}^{n+1}=\left(\theta_{0}^{n+1}, \theta_{1}^{n+1}, \ldots, \theta_{M_{1}+1}^{n+1}\right)^{T}
$$

is an $M_{1}+2$ dimensional vector and $A$ is an $\left(M_{1}+2\right) \times\left(M_{1}+2\right)$ tridiagonal matrix with entries

$$
\begin{gathered}
a_{0,0}=1 ; a_{M_{1}+2, M_{1}+2}=-k_{1} ; a_{j, j}=1+a_{1}^{2}+2 \frac{\Delta t}{\Delta x^{2}}, j=1,2, \ldots, M_{1} \\
a_{j, j+1}=-\frac{\Delta t}{\Delta x^{2}}, j=0,1, \ldots, M_{1} ; a_{j+1, j}=-\frac{\Delta t}{\Delta x^{2}}, j=0,1, \ldots, M_{1}-1 ; \\
a_{M_{1}, M_{1}+1}=k_{1}-k \Delta x .
\end{gathered}
$$

$B$ is an $M_{1}+2$ dimensional vector with entries

$$
\begin{gathered}
B_{0}=0, B_{M_{1}+1}=-k \Delta x \psi_{M_{2}}^{n-1}, \\
B_{j}=\left(1+a_{1}^{2}\right) \theta_{j}^{n}+\alpha_{1}\left(g\left(\theta^{n}, \psi^{n}\right)-g\left(\theta^{n-1}, \psi^{n-1}\right)\right), j=1,2, \ldots, M_{1} .
\end{gathered}
$$

By (2.1), $A$ is a diagonally dominant matrix and so is invertible by the Gerschgorin Theorem. Therefore, $\left\{\theta_{j}^{n}\right\}$ can be advanced to $\left\{\theta_{j}^{n+1}\right\}$.

We now give the energy estimates in the next two theorems.

Theorem 2.2. Assume that schemes (1.13)-(1.20) have been solved to $t^{n}\left(t^{n} \geq \Delta t\right)$. If $a_{1}, a_{2}, \alpha_{1}, \alpha_{2}$ are small enough, $\Delta x, \Delta t$ are chosen such that they satisfy (2.1) and

$$
\begin{gathered}
\Delta t \leq \Delta x^{1+p}, \text { for some constant } p, 0 \leq p \leq 1, \\
k \Delta x^{p}+2\left(a_{1}+a_{2}\right)\left(\frac{k_{1} \alpha_{1}^{2}}{1+a_{1}^{2}}+\frac{k_{2} \alpha_{2}^{2}}{d+a_{2}^{2}}\right) \leq \frac{1}{2} \min \left\{k_{1}\left(1+a_{1}^{2}\right), k_{2}\left(d+a_{2}^{2}\right)\right\},
\end{gathered}
$$

then there exists a constant $C$, which satisfies

$$
C \leq \frac{\max \left\{k_{1}, k_{2}, k\right\}}{\min \left\{\frac{1}{2} k_{1}\left(1+a_{1}^{2}\right), \frac{1}{2} k_{2}\left(d+a_{2}^{2}\right), \frac{k_{1}}{1+a_{1}^{2}}, \frac{k_{2}}{d+a_{2}^{2}}, k_{1}, k_{2}, k\right\}},
$$

such that

$$
\begin{aligned}
& \sum_{j=0}^{M_{1}-1}\left(\delta \theta_{j+1 / 2}^{n}\right)^{2} \Delta x+\sum_{j=M_{2}}^{M-1}\left(\delta \psi_{j+1 / 2}^{n}\right)^{2} \Delta x+\sum_{q=0}^{n} \sum_{j=0}^{M_{1}}\left(\delta^{2} \theta_{j}^{q}\right)^{2} \Delta x \Delta t \\
& \quad+\sum_{q=0}^{n} \sum_{j=M_{2}}^{M}\left(\delta^{2} \psi_{j}^{q}\right)^{2} \Delta x \Delta t+\sum_{q=0}^{n-1} \sum_{j=0}^{M_{1}}\left(\frac{\theta_{j}^{q+1}-\theta_{j}^{q}}{\Delta t}\right)^{2} \Delta x \Delta t\left(\frac{\psi_{j}^{q-1}-\psi_{j}^{q}}{\Delta t}\right)^{2} \Delta x \Delta t \\
& \quad+\sum_{q=0}^{n-1} \sum_{j=M_{2}}^{M}+\left(\theta_{M_{1}}^{n}-\psi_{M_{2}}^{n}\right)^{2} \leq C C_{0}
\end{aligned}
$$

Remark 2.1. (2.10) is not the CFL condition, it is weaker than the CFL condition. The optimal error bound, which will be given in Theorem 4.4., will depend on $p$. 
Proof. We square both sides of (1.13) to get

$$
\begin{aligned}
\left(1+a_{1}^{2}\right)^{2}\left(\frac{\theta_{j}^{q+1}-\theta_{j}^{q}}{\Delta t}\right)^{2}+\left(\delta^{2} \theta_{j}^{q+1}\right)^{2}-2(1 & \left.+a_{1}^{2}\right) \delta^{2} \theta_{j}^{q+1}\left(\frac{\theta_{j}^{q+1}-\theta_{j}^{q}}{\Delta t}\right) \\
& =\alpha_{1}^{2}\left(\frac{g\left(\theta^{q}, \psi^{q}\right)-g\left(\theta^{q-1}, \psi^{q-1}\right)}{\Delta t}\right)^{2} .
\end{aligned}
$$

We then multiply both sides of (2.13) by $\frac{k_{1}}{1+a_{1}^{2}} \Delta x \Delta t$, sum over $q, j$ to get

$$
\begin{aligned}
k_{1}(1 & \left.+a_{1}^{2}\right) \sum_{q=0}^{n-1} \sum_{j=0}^{M_{1}}\left(\frac{\theta_{j}^{q+1}-\theta_{j}^{q}}{\Delta t}\right)^{2} \Delta x \Delta t+\frac{k_{1}}{1+a_{1}^{2}} \sum_{q=1}^{n} \sum_{j=0}^{M_{1}}\left(\delta^{2} \theta_{j}^{q+1}\right)^{2} \Delta x \Delta t \\
& -2 k_{1} \sum_{q=0}^{n-1} \sum_{j=0}^{M_{1}}\left(\frac{\theta_{j}^{q+1}-\theta_{j}^{q}}{\Delta t}\right)\left(\delta^{2} \theta_{j}^{q+1}\right) \Delta x \Delta t \\
= & \frac{k_{1} \alpha_{1}^{2}}{1+a_{1}^{2}} \sum_{q=0}^{n-1} \sum_{j=0}^{M_{1}}\left(\frac{g\left(\theta^{q}, \psi^{q}\right)-g\left(\theta^{q-1}, \psi^{q-1}\right)}{\Delta t}\right)^{2} \Delta x \Delta t .
\end{aligned}
$$

We then apply the summation by parts formula to the third term on the left-hand side of (2.14) to get

$$
\begin{aligned}
- & 2 k_{1} \sum_{q=0}^{n-1} \sum_{j=0}^{M_{1}}\left(\frac{\theta_{j}^{q+1}-\theta_{j}^{q}}{\Delta t}\right)\left(\delta^{2} \theta_{j}^{q+1}\right) \Delta x \Delta t \\
= & 2 k_{1} \sum_{q=0}^{n-1} \sum_{j=0}^{M_{1}-1} \frac{\delta \theta_{j+1 / 2}^{q+1}-\delta \theta_{j+1 / 2}^{q}}{\Delta t}\left(\delta \theta_{j+1 / 2}^{q+1}\right) \Delta x \Delta t \\
& -2 k_{1} \sum_{q=0}^{n-1} \frac{\theta_{M_{1}}^{q+1}-\theta_{M_{1}}^{q}}{\Delta t} \delta \theta_{M_{1}+1 / 2}^{q+1} \Delta t+2 k_{1} \sum_{q=0}^{n-1} \frac{\theta_{0}^{q+1}-\theta_{0}^{q}}{\Delta t} \delta \theta_{-1 / 2}^{q+1} \Delta t .
\end{aligned}
$$

The last term of (2.15) is zero by the first part of the boundary condition (1.17). We use the second part of (1.17) to the middle term and simplify the first term on the right-hand side of (2.15) to get

$$
\begin{aligned}
-2 k_{1} \sum_{q=0}^{n-1} \sum_{j=0}^{M_{1}} \frac{\theta_{j}^{q+1}-\theta_{j}^{q}}{\Delta t}\left(\delta^{2} \theta_{j}^{q+1}\right) \Delta x \Delta t \geq k_{1} \sum_{j=0}^{M_{1}-1}\left(\delta \theta_{j+1 / 2}^{n}\right)^{2} \Delta x \\
\quad-k_{1} \sum_{j=0}^{M_{1}-1}\left(\delta \theta_{j+1 / 2}^{0}\right)^{2} \Delta x-2 k \sum_{q=0}^{n-1} \frac{\theta_{M_{1}}^{q+1}-\theta_{M_{1}}^{q}}{\Delta t}\left(\theta_{M_{1}}^{q+1}-\psi_{M_{2}}^{q}\right) \Delta t .
\end{aligned}
$$

To estimate the term on the right-hand side of (2.14), we use the fact that, for any number $r$,

$$
\max \{r, 0\}=\frac{1}{2} r+\frac{1}{2}|r|,
$$


by using the definition of $g(\theta, \psi)$ and (2.17), we get

$$
\begin{aligned}
& \frac{g\left(\theta_{j}^{q}, \psi_{j}^{q}\right)-g\left(\theta_{j}^{q-1}, \psi_{j}^{q-1}\right)}{\Delta t} \\
& =\left(\max \left\{a_{1} \sum_{j=0}^{M_{1}} \theta_{j}^{q} \Delta x+a_{2} \sum_{j=M_{2}}^{M} \psi_{j}^{q}-\tilde{g} ; 0\right\}\right. \\
& \left.-\max \left\{a_{1} \sum_{j=0}^{M_{1}} \theta_{j}^{q-1} \Delta x+a_{2} \sum_{j=M_{2}}^{M} \psi_{j}^{q-1} \Delta x-\tilde{g} ; 0\right\}\right) / \Delta t \\
& =\frac{a_{1}}{2} \sum_{j=0}^{M_{1}} \frac{\theta_{j}^{q}-\theta_{j}^{q-1}}{\Delta t} \Delta x+\frac{a_{2}}{2} \sum_{j=M_{2}}^{M} \frac{\psi_{j}^{q}-\psi_{j}^{q-1}}{\Delta t} \Delta x \\
& +\frac{1}{2}\left(\left|a_{1} \sum_{j=0}^{M_{1}} \theta_{j}^{q} \Delta x+a_{2} \sum_{j=M_{2}}^{M} \psi_{j}^{q} \Delta x-\tilde{g}\right|\right. \\
& \left.-\left|a_{1} \sum_{j=0}^{M_{1}} \theta_{j}^{q-1} \Delta x+a_{2} \sum_{j=M_{2}}^{M} \psi_{j}^{q-1} \Delta x-\tilde{g}\right|\right) / \Delta t,
\end{aligned}
$$

we then use a basic inequality ||$a|-| b|| \leq|a-b|$ to (2.18) and get

$$
\begin{aligned}
& \left|\frac{g\left(\theta_{j}^{q}, \psi_{j}^{q}\right)-g\left(\theta_{j}^{q-1}, \psi_{j}^{q-1}\right)}{\Delta t}\right| \\
& \quad \leq \frac{a_{1}}{2} \sum_{j=0}^{M_{1}}\left|\frac{\theta_{j}^{q}-\theta_{j}^{q-1}}{\Delta t}\right| \Delta x+\frac{a_{2}}{2} \sum_{j=M_{2}}^{M}\left|\frac{\psi_{j}^{q}-\psi_{j}^{q-1}}{\Delta t}\right| \Delta x \\
& \quad+\frac{1}{2}\left|a_{1} \sum_{j=0}^{M_{1}} \frac{\theta_{j}^{q}-\theta_{j}^{q-1}}{\Delta t} \Delta x+a_{2} \sum_{j=M_{2}}^{M} \frac{\psi_{j}^{q}-\psi_{j}^{q-1}}{\Delta t} \Delta x\right| \Delta x+a_{2} \sum_{j=M_{2}}^{M}\left|\frac{\psi_{j}^{q}-\psi_{j}^{q-1}}{\Delta t}\right| \Delta x .
\end{aligned}
$$

So,

$$
\begin{aligned}
& \left(\frac{g\left(\theta_{j}^{q}, \psi_{j}^{q}\right)-g\left(\theta_{j}^{q-1}, \psi_{j}^{q-1}\right)}{\Delta t}\right)^{2} \\
& \quad \leq 2 a_{1}^{2}\left(\sum_{j=0}^{M_{1}}\left|\frac{\theta_{j}^{q}-\theta_{j}^{q-1}}{\Delta t}\right| \Delta x\right)^{2}+2 a_{2}^{2}\left(\sum_{j=M_{2}}^{M}\left|\frac{\psi_{j}^{q}-\psi_{j}^{q-1}}{\Delta t}\right| \Delta x\right)^{2} \\
& \leq 2 a_{1}^{2}\left(\sum_{j=0}^{M_{1}} \Delta x\right)\left(\sum_{j=0}^{M_{1}}\left(\frac{\theta_{j}^{q}-\theta_{j}^{q-1}}{\Delta t}\right)^{2} \Delta x\right)
\end{aligned}
$$




$$
\begin{aligned}
& +2 a_{2}^{2}\left(\sum_{j=M_{2}}^{M} \Delta x\right)\left(\sum_{j=M_{2}}^{M}\left(\frac{\psi_{j}^{q}-\psi_{j}^{q-1}}{\Delta t}\right)^{2} \Delta x\right) \\
= & 2 l_{1} a_{1}^{2}\left(\sum_{j=0}^{M_{1}}\left(\frac{\theta_{j}^{q}-\theta_{j}^{q-1}}{\Delta t}\right)^{2} \Delta x\right)+2 l_{2} a_{2}^{2}\left(\sum_{j=M_{2}}^{M}\left(\frac{\psi_{j}^{q}-\psi_{j}^{q-1}}{\Delta t}\right)^{2} \Delta x\right) .
\end{aligned}
$$

Therefore,

$$
\begin{aligned}
& \frac{k_{1} \alpha_{1}^{2}}{1+a_{1}^{2}} \sum_{q=0}^{n-1} \sum_{j=0}^{M_{1}}\left(\frac{g\left(\theta_{j}^{q}, \psi_{j}^{q}\right)-g\left(\theta_{j}^{q-1}, \psi_{j}^{q-1}\right)}{\Delta t}\right)^{2} \Delta x \Delta t \\
& \leq \frac{k_{1} \alpha_{1}^{2}}{1+a_{1}^{2}}\left(2 l_{1}^{2} a_{1}^{2} \sum_{q=0}^{n-1} \sum_{j=0}^{M_{1}}\left(\frac{\theta_{j}^{q}-\theta_{j}^{q-1}}{\Delta t}\right)^{2} \Delta x \Delta t\right. \\
& \left.+2 l_{2}^{2} a_{2}^{2} \sum_{q=0}^{n-1} \sum_{j=M_{2}}^{M}\left(\frac{\psi_{j}^{q}-\psi_{j}^{q-1}}{\Delta t}\right)^{2} \Delta x \Delta t\right) \\
& \leq \frac{2 k_{1} \alpha_{1}^{2}\left(a_{1}^{2}+a_{2}^{2}\right)}{1+a_{1}^{2}}\left(\sum_{q=0}^{n-1} \sum_{j=0}^{M_{1}}\left(\frac{\theta_{j}^{q}-\theta_{j}^{q-1}}{\Delta t}\right)^{2} \Delta x \Delta t\right. \\
& \left.+\sum_{q=0}^{n-1} \sum_{j=M_{2}}^{M}\left(\frac{\psi_{j}^{q}-\psi_{j}^{q-1}}{\Delta t}\right)^{2} \Delta x \Delta t\right) .
\end{aligned}
$$

If we put (2.16) and (2.21) back to (2.14), we then get

$$
\begin{aligned}
& k_{1}\left(1+a_{1}^{2}\right) \sum_{q=0}^{n-1} \sum_{j=0}^{M_{1}}\left(\frac{\theta_{j}^{q+1}-\theta_{j}^{q}}{\Delta t}\right)^{2} \Delta x \Delta t+\frac{k_{1}}{1+a_{1}^{2}} \sum_{q=1}^{n} \sum_{j=0}^{M_{1}}\left(\delta^{2} \theta_{j}^{q}\right)^{2} \Delta x \Delta t \\
& +k_{1} \sum_{j=0}^{M_{1}-1}\left(\delta \theta_{j+1 / 2}^{n}\right)^{2} \Delta x-2 k \sum_{q=0}^{n-1}\left(\frac{\theta_{M}^{q+1}-\theta_{M_{1}}^{q}}{\Delta t}\right)\left(\theta_{M_{1}}^{q+1}-\psi_{M_{2}}^{q}\right) \Delta t \\
& \leq k_{1} \sum_{j=0}^{M_{1}-1}\left(\delta \theta_{j+1 / 2}^{0}\right)^{2} \Delta x+\frac{2 k_{1}\left(a_{1}+a_{2}\right) \alpha_{1}^{2}}{1+a_{1}^{2}}\left(\sum_{q=0}^{n-1} \sum_{j=0}^{M_{1}}\left(\frac{\theta_{j}^{q+1}-\theta_{j}^{q}}{\Delta t}\right)^{2} \Delta x \Delta t\right. \\
& \left.\quad+\sum_{q=0}^{n-1} \sum_{j=M_{2}}^{M}\left(\frac{\psi_{j}^{q+1}-\psi_{j}^{q}}{\Delta t}\right)^{2} \Delta x \Delta t\right) .
\end{aligned}
$$


We obtain similar estimates to (1.14) and combine with (2.22) to get

$$
\begin{aligned}
k_{1}(1 & \left.+a_{1}^{2}\right) \sum_{q=0}^{n-1} \sum_{j=0}^{M_{1}}\left(\frac{\theta_{j}^{q+1}-\theta_{j}^{q}}{\Delta t}\right)^{2} \Delta x \Delta t+k_{2}\left(d+a_{2}^{2}\right) \sum_{q=0}^{n-1} \sum_{j=M_{2}}^{M}\left(\frac{\psi_{j}^{q+1}-\psi_{j}^{q}}{\Delta t}\right)^{2} \Delta x \Delta t \\
& +\frac{k_{1}}{1+a_{1}^{2}} \sum_{q=1}^{n} \sum_{j=0}^{M_{1}}\left(\delta^{2} \theta_{j}^{q}\right)^{2} \Delta x \Delta t+\frac{k_{2}}{d+a_{2}^{2}} \sum_{q=1}^{n} \sum_{j=M_{2}}^{M}\left(\delta^{2} \psi_{j}^{q}\right)^{2} \Delta x \Delta t \\
& +k_{1} \sum_{j=0}^{M_{1}-1}\left(\delta \theta_{j+1 / 2}^{n}\right)^{2} \Delta x+k_{2} \sum_{j=M_{2}}^{M-1}\left(\delta \psi_{j+1 / 2}^{n}\right)^{2} \Delta x+2 k \sum_{q=0}^{n-1}\left(\frac{\theta_{M_{1}}^{q+1}-\theta_{M_{1}}^{q}}{\Delta t}\right)^{2} \\
& \times\left(\theta_{M_{1}}^{q+1}-\psi_{M_{2}}^{q}\right) \Delta t-2 k \sum_{q=0}^{n-1}\left(\frac{\psi_{M_{2}}^{q+1}-\psi_{M_{2}}^{q}}{\Delta t}\right)\left(\theta_{M_{1}}^{q+1}-\psi_{M_{2}}^{q+1}\right) \Delta t \\
\leq & k_{1} \sum_{j=0}^{M_{1}-1}\left(\delta \theta_{j+1 / 2}^{0}\right)^{2} \Delta x+k_{2} \sum_{j=M_{2}}^{M-1}\left(\delta \psi_{j+1 / 2}^{0}\right)^{2} \Delta x+2\left(a_{1}+a_{2}\right)\left(\frac{k_{1} \alpha_{1}^{2}}{1+a_{1}^{2}}+\frac{k_{2} \alpha_{2}^{2}}{d+a_{2}^{2}}\right) \\
& \times\left(\sum_{q=0}^{n-1} \sum_{j=0}^{M_{1}}\left(\frac{\theta_{j}^{q+1}-\theta_{j}^{q}}{\Delta t}\right)^{2} \Delta x \Delta t+\sum_{q=0}^{n-1} \sum_{j=M_{2}}^{M}\left(\frac{\psi_{j}^{q+1}-\psi_{j}^{q}}{\Delta t}\right)^{2} \Delta x \Delta t\right) .
\end{aligned}
$$

The two terms just before the inequality sign in (2.23) can be estimated by

$$
\begin{aligned}
2 k & \sum_{q=0}^{n-1}\left(\frac{\theta_{M_{1}}^{q+1}-\theta_{M_{1}}^{q}}{\Delta t}\right)\left(\theta_{M_{1}}^{q+1}-\psi_{M_{2}}^{q}\right) \Delta t-2 k \sum_{q=0}^{n-1}\left(\frac{\psi_{M_{2}}^{q+1}-\psi_{M_{2}}^{q}}{\Delta t}\right)\left(\theta_{M_{1}}^{q+1}-\psi_{M_{2}}^{q+1}\right) \Delta t \\
= & 2 k \sum_{q=0}^{n-1}\left[\frac{\theta_{M_{1}}^{q+1}-\theta_{M_{1}}^{q}}{\Delta t}\left(\theta_{M_{1}}^{q+1}-\psi_{M_{2}}^{q+1}\right)-\frac{\psi_{M_{2}}^{q+1}-\psi_{M_{2}}^{q}}{\Delta t}\left(\theta_{M_{1}}^{q+1}-\psi_{M_{2}}^{q+1}\right)\right] \Delta t \\
& +2 k \Delta t \sum_{q=0}^{n-1} \frac{\theta_{M_{1}}^{q+1}-\theta_{M_{1}}^{q}}{\Delta t} \frac{\psi_{M_{2}}^{q+1}-\psi_{M_{2}}^{q} \Delta t}{\Delta t} \Delta \\
= & 2 k \sum_{q=0}^{n-1}\left(\theta_{M_{1}}^{q+1}-\psi_{M_{2}}^{q+1}\right)\left(\left(\theta_{M_{1}}^{q+1}-\psi_{M_{2}}^{q+1}\right)-\left(\theta_{M_{1}}^{q}-\psi_{M_{2}}^{q}\right)\right) \\
& +2 k \Delta t \sum_{q=0}^{n-1} \frac{\theta_{M_{1}}^{q+1}-\theta_{M_{1}}^{q}}{\Delta t} \frac{\psi_{M_{2}}^{q+1}-\psi_{M_{2}}^{q} \Delta t}{\Delta t} \\
\geq & \left.2 k \sum_{q=0}^{n-1}\left(\theta_{M_{1}}^{q+1}-\psi_{M_{2}}^{q+1}\right)^{2}-k \sum_{q=0}^{n-1}\left(\theta_{M_{1}}^{q+1}-\psi_{M_{2}}^{q+1}\right)^{2}+\sum_{q=0}^{n-1}\left(\theta_{M_{1}}^{q}-\psi_{M_{2}}^{q}\right)^{2}\right] \\
& -k \Delta t \sum_{q=0}^{n-1}\left[\left(\frac{\theta_{M_{1}}^{q+1}-\theta_{M_{1}}^{q}}{\Delta t}\right)^{2}+\left(\frac{\psi_{M_{2}}^{q+1}-\psi_{M_{2}}^{q}}{\Delta t}\right)^{2}\right] \Delta t \\
\geq & k\left(\theta_{M_{1}}^{n}-\psi_{M_{2}}^{n}\right)^{2}-k\left(\theta_{M_{1}}^{0}-\psi_{M_{2}}^{0}\right)^{2} \\
& -k \Delta t \sum_{q=0}^{n-1}\left[\left(\frac{\theta_{M_{1}}^{q+1}-\theta_{M_{1}}^{q}}{\Delta t}\right)^{2}+\left(\frac{\psi_{M_{2}}^{q+1}-\psi_{M_{2}}^{q}}{\Delta t}\right)^{2}\right] \Delta t[\mathrm{by} \mathrm{using} \mathrm{(2.9)]}
\end{aligned}
$$




$$
\begin{aligned}
= & k\left(\theta_{M_{1}}^{n}-\psi_{M_{2}}^{n}\right)^{2}-k\left(\theta_{M_{1}}^{0}-\psi_{M_{2}}^{0}\right)^{2} \\
& -k \Delta x^{p} \sum_{q=0}^{n-1}\left[\left(\frac{\theta_{M_{1}}^{q+1}-\theta_{M_{1}}^{q}}{\Delta t}\right)^{2}+\left(\frac{\psi_{M_{2}}^{q+1}-\psi_{M_{2}}^{q}}{\Delta t}\right)^{2}\right] \Delta x \Delta t .
\end{aligned}
$$

If we put (2.24) back to (2.23), we then get

$$
\begin{aligned}
k_{1}(1+ & \left.a_{1}^{2}\right) \sum_{q=0}^{n-1} \sum_{j=0}^{M_{1}}\left(\frac{\theta_{j}^{q+1}-\theta_{j}^{q}}{\Delta t}\right)^{2} \Delta x \Delta t+k_{2}\left(d+a_{2}^{2}\right) \sum_{q=0}^{n-1} \sum_{j=M_{2}}^{M}\left(\frac{\psi_{j}^{q+1}-\psi_{j}^{q}}{\Delta t}\right)^{2} \Delta x \Delta t \\
& +\frac{k_{1}}{1+a_{1}^{2}} \sum_{q=1}^{n} \sum_{j=0}^{M_{1}}\left(\delta^{2} \theta_{j}^{q}\right)^{2} \Delta x \Delta t+\frac{k_{2}}{d+a_{2}^{2}} \sum_{q=1}^{n} \sum_{j=M_{2}}^{M}\left(\delta^{2} \psi_{j}^{q}\right)^{2} d x d t \\
& +k_{1} \sum_{j=0}^{M_{1}-1}\left(\delta \theta_{j+1 / 2}^{n}\right)^{2} \Delta x+k_{2} \sum_{j=M_{2}}^{M-1}\left(\delta \psi_{j+1 / 2}^{n}\right)^{2} \Delta x+k\left(\theta_{M_{1}}^{n}-\psi_{M_{2}}^{n}\right)^{2} \\
\leq & k_{1} \sum_{j=0}^{M_{1}-1}\left(\delta \theta_{j+1 / 2}^{0}\right)^{2} \Delta x+k_{2} \sum_{j=M_{2}}^{M-1}\left(\delta \psi_{j+1 / 2}^{0}\right)^{2} \Delta x+k\left(\theta_{M_{1}}^{0}-\psi_{M_{2}}^{0}\right)^{2} \\
& +\left(k \Delta x^{p}+2\left(a_{1}+a_{2}\right)\left(\frac{k_{1} \alpha_{1}^{2}}{1+a_{1}^{2}}+\frac{k_{2} \alpha_{2}^{2}}{d+a_{2}^{2}}\right)\right) \\
& \times\left(\sum_{q=0}^{n-1} \sum_{j=0}^{M_{1}}\left(\frac{\theta_{j}^{q+1}-\theta_{j}^{q}}{\Delta t}\right)^{2} \Delta x \Delta t+\sum_{q=0}^{n-1} \sum_{j=M_{2}}^{M}\left(\frac{\psi_{j}^{q+1}-\psi_{j}^{q}}{\Delta t}\right)^{2} \Delta x \Delta t\right)
\end{aligned}
$$

(2.12) follows from (2.25) by using (2.10).

By Using Theorem 2.2, we can get a few more estimates, given in the next theorem.

Theorem 2.3. Assume that schemes (1.13)-(1.20) have been solved to $t^{n}\left(t^{n} \geq \Delta t\right)$, and $C$ is the constant as described in Theorem 2.2. If $a_{1}, a_{2}, \alpha_{1}, \alpha_{2}$ are small enough and $\Delta x, \Delta t$ are chosen to satisfy (2.1), (2.10), and (2.11), then

$$
\sum_{j=0}^{M_{1}} \theta_{j}^{n 2} \Delta x+\sum_{j=M_{2}}^{M} \psi_{j}^{n 2} \Delta x \leq C C_{0},
$$

and

$$
\begin{aligned}
\sum_{j=1}^{M_{1}}\left(\delta^{2} \theta_{j}^{n}\right)^{2} \Delta x+\sum_{j=M_{2}+1}^{M}\left(\delta^{2} \psi_{j}^{n}\right)^{2} \Delta x & +\sum_{q=0}^{n-1} \sum_{j=0}^{M_{1}}\left(\frac{\delta \theta_{j+1 / 2}^{q+1}-\delta \theta_{j+1 / 2}^{q}}{\Delta t}\right)^{2} \Delta x \Delta t \\
& +\sum_{q=0}^{n-1} \sum_{j=M_{2}}^{M}\left(\frac{\delta \psi_{j+1 / 2}^{q+1}-\delta \psi_{j+1 / 2}^{q}}{\Delta t}\right)^{2} \Delta x \Delta t \leq C C_{0} .
\end{aligned}
$$

Proof. By using the first part of (1.17), we have

$$
\theta_{j}^{n}-\theta_{0}^{n}=\theta_{j}^{n}=\sum_{l=0}^{j-1} \delta \theta_{j+1 / 2}^{n} \Delta x,
$$


we then square both sides of (2.28), and use the Cauchy-Schwarz inequality to get

$$
\begin{aligned}
\theta_{j}^{n 2} & =\left(\sum_{l=0}^{j-1} \delta \theta_{l+1 / 2}^{n} \Delta x\right)^{2} \leq\left(\sum_{l=0}^{j-1} \Delta x\right)\left(\sum_{l=0}^{j-1} \delta \theta_{l+1 / 2}^{n 2} \Delta x\right) \\
& \leq l_{1}\left(\sum_{l=0}^{j-1} \delta \theta_{l+1 / 2}^{n 2} \Delta x\right) \leq l_{1}\left(\sum_{j=0}^{M_{1}-1} \delta \theta_{j}^{n 2} \Delta x\right),
\end{aligned}
$$

we then multiply both sides of (2.29) by $\Delta x$, sum over $j$, and use (2.12) to get

$$
\sum_{j=0}^{M-1} \theta_{j}^{n 2} \Delta x \leq l_{1}\left(\sum_{j=0}^{M_{1}} \Delta x\right)\left(\sum_{j=0}^{M_{1}} \delta \theta_{j}^{n 2} \Delta x\right) \leq l_{1}^{2}\left(\sum_{j=0}^{M_{1}-1} \delta \theta_{j}^{n 2} \Delta x\right) \leq C C_{0},
$$

the other term in (2.26) can be proved similarly, this proves (2.26).

To prove (2.27), we apply the $\delta$ operator to both sides of (1.13), notice that the right-hand side of (1.13) does not depend on $j$, so we get

$$
\left(1+a_{1}^{2}\right) \frac{\delta \theta_{j+1 / 2}^{q+1}-\delta \theta_{j+1 / 2}^{q}}{\Delta t}-\delta^{3} \theta_{j+1 / 2}^{q+1}=0
$$

We then multiply both sides of (2.31) by $\frac{\delta \theta_{j+1 / 2}^{q+1}-\delta \theta_{j+1 / 2}^{q}}{\Delta t} \Delta x \Delta t$, sum over $q, j$ and use (1.17) to get

$$
\begin{aligned}
(1+ & \left.a_{1}^{2}\right) \sum_{q=0}^{n-1} \sum_{j=0}^{M_{1}}\left(\frac{\delta \theta_{j+1 / 2}^{q+1}-\delta \theta_{j+1 / 2}^{q}}{\Delta t}\right)^{2} \Delta x \Delta t+\sum_{q=0}^{n-1} \sum_{j=1}^{M_{1}} \delta^{2} \theta_{j}^{q+1}\left(\delta^{2} \theta_{j}^{q+1}-\delta^{2} \theta_{j}^{q}\right) \Delta x \\
& +\sum_{q=0}^{n-1} \delta^{2} \theta_{0}^{q+1} \frac{\delta \theta_{1 / 2}^{q+1}-\delta \theta_{1 / 2}^{q}}{\Delta t} \Delta t-\sum_{q=0}^{n-1} \delta^{2} \theta_{M_{1}+1}^{q+1} \frac{\delta \theta_{M_{1}+1 / 2}^{q+1}-\delta \theta_{M_{1}+1 / 2}^{q}}{\Delta t} \Delta t=0 .
\end{aligned}
$$

By (1.20),

$$
\delta^{2} \theta_{0}^{q+1}=\frac{\theta_{1}^{q+1}-2 \theta_{0}^{q+1}+\theta_{-1}^{q+1}}{\Delta x^{2}}=0
$$

so the third term of (2.32) vanishes. We assume the schemes are extended, so that

$$
\delta^{2} \theta_{M_{1}+1}^{q+1}=\frac{\theta_{M_{1}+2}^{q+1}-2 \theta_{M_{1}+1}^{q+1}+\theta_{M_{1}}^{q+1}}{\Delta x^{2}}=0,
$$

so the last term on the right-hand side of (2.32) also vanishes. We now estimate the second term on the right-hand side of (2.32) by

$$
\begin{aligned}
\sum_{q=0}^{n-1} \sum_{j=1}^{M_{1}}\left(\left(\delta^{2} \theta_{j}^{q+1}\right)^{2}-\delta^{2} \theta_{j}^{q+1} \delta^{2} \theta_{j}^{q}\right) \Delta x & \geq \frac{1}{2} \sum_{q=0}^{n-1} \sum_{j=1}^{M_{1}}\left(\delta^{2} \theta_{j}^{q+1}\right)^{2} \Delta x-\frac{1}{2} \sum_{q=0}^{n-1} \sum_{j=1}^{M_{1}}\left(\delta \theta_{j}^{q}\right)^{2} \Delta x \\
& \geq \frac{1}{2} \sum_{j=1}^{M_{1}}\left(\delta^{2} \theta_{j}^{n}\right)^{2} \Delta x-\frac{1}{2} \sum_{j=1}^{M_{1}}\left(\delta^{2} \theta_{j}^{0}\right)^{2} \Delta x
\end{aligned}
$$


So (2.32) becomes

$$
\begin{array}{r}
\frac{1}{2} \sum_{j=1}^{M_{1}-1}\left(\delta^{2} \theta_{j}^{n}\right)^{2} \Delta x+\left(1+a_{1}^{2}\right) \sum_{q=0}^{n-1} \sum_{j=0}^{M_{1}}\left(\frac{\delta \theta_{j+1 / 2}^{q+1}-\delta \theta_{j+1 / 2}^{q}}{\Delta t}\right)^{2} \Delta x \Delta t \\
\quad \leq \frac{1}{2} \sum_{j=1}^{M_{1}-1}\left(\delta^{2} \theta_{j}^{0}\right)^{2} \Delta x \leq \frac{1}{2} C_{0} .
\end{array}
$$

The other terms of (2.27) about $\psi_{j}^{q}$ can be estimated similarly.

\section{WEAK TRUNCATION ERRORS}

In this section, we will obtain the truncation errors to schemes (1.13)-(1.20). Let $h=(\Delta x, \Delta t)$, we first construct the approximate solutions $\left\{\theta^{h}, \psi^{h}, g^{h}\right\}$, which are linear interpolations of $\left\{\theta_{j}^{q}\right\},\left\{\psi_{j}^{q}\right\}$, and $\left\{g\left(\theta_{j}^{q}, \psi_{j}^{q}\right)\right\}$.

$$
\begin{aligned}
\theta^{h}(x, t)= & \theta_{j}^{q}+\frac{\theta_{j}^{q+1}-\theta_{j}^{q}}{\Delta t}\left(t-t^{q}\right)+\delta \theta_{j+1 / 2}^{q}\left(x-x_{j}\right) \\
& +\frac{\delta \theta_{j+1 / 2}^{q+1}-\delta \theta_{j+1 / 2}^{q}}{\Delta t}\left(x-x_{j}\right)\left(t-t^{q}\right),(x, t) \in\left[x_{j}, x_{j+1}\right] \\
& \times\left(t^{q}, t^{q+1}\right], 0 \leq j \leq M_{1}, q \geq 0, \\
\psi^{h}(x, t)= & \psi_{j}^{q-1}+\frac{\psi_{j}^{q}-\psi_{j}^{q-1}}{\Delta t}\left(t-t^{q}\right)+\delta \psi_{j+1 / 2}^{q-1}\left(x-x_{j}\right) \\
& +\frac{\delta \psi_{j+1 / 2}^{q}-\delta \psi_{j+1 / 2}^{q-1}}{\Delta t}\left(x-x_{j}\right)\left(t-t^{q}\right),(x, t) \in\left[x_{j}, x_{j+1}\right] \\
& \times\left(t^{q}, t^{q+1}\right], M_{2}-1 \leq j \leq M-1, q \geq 0,
\end{aligned}
$$

and

$$
g^{h}(t)=g\left(\theta^{q}, \psi^{q}\right)+\frac{g\left(\theta^{q+1}, \psi^{q+1}\right)-g\left(\theta^{q}, \psi^{q}\right)}{\Delta t}\left(t-t^{q}\right), t \in\left(t^{q}, t^{q+1}\right], q \geq 0 .
$$

Notice that, with the above constructions, we can obtain the following boundary conditions to $\theta^{h}, \psi^{h}$ :

$$
\begin{aligned}
& \theta^{h}(0, t)=0,-k_{1} \theta_{x}^{h}\left(l_{1}, t\right)=k\left(\theta^{h}\left(l_{1}, t\right)-\psi^{h}\left(l_{2}, t\right)\right), \\
& \psi^{h}(1, t)=0,-k_{2} \psi_{x}^{h}\left(l_{2}, t\right)=k\left(\theta^{h}\left(l_{1}, t\right)-\psi^{h}\left(l_{2}, t\right)\right) .
\end{aligned}
$$

Both functions $\theta^{h}, \psi^{h}$ are constructed slightly beyond their ranges $\left[0, l_{1}\right]$ and $\left[l_{2}, 1\right]$ in order for us to take care of the boundary conditions. A direct result of Theorems 2.2, 2.3 will give the following theorem. 
Theorem 3.1. Let $\left\{\theta^{h}, \psi^{h}\right\}$ be the approximate solutions constructed in (3.1), (3.2), $g^{h}(t)$ be the function given in (3.3). Then for any $T>0$,

$$
\begin{aligned}
& \left\|\theta^{h}(x, t)\right\|_{H^{1}\left(0, l_{1}\right)}+\left\|\theta^{h}(x, t)\right\|_{H^{1}\left(\left(0, l_{1}\right) \times(0, T)\right)}+\left\|\theta_{x t}^{h}(x, t)\right\|_{L^{2}\left(\left(0, l_{1}\right) \times(0, T)\right)} \\
& \quad+\left\|\theta^{h}\left(l_{1}, t\right)\right\|_{H^{1}(0, T)}+\left\|\psi^{h}(x, t)\right\|_{H^{1}\left(l_{2}, 1\right)}+\left\|\psi^{h}(x, t)\right\|_{H^{1}\left(\left(l_{2}, 1\right) \times(0, T)\right)} \\
& \quad+\left\|\psi_{x t}^{h}(x, t)\right\|_{L^{2}\left(\left(l_{2}, 1\right) \times(0, T)\right)}+\left\|\psi^{h}\left(l_{2}, t\right)\right\|_{H^{1}(0, T)}+\left\|g^{h}(t)\right\|_{l^{2}(0, T)} \leq C C_{0}^{1 / 2},
\end{aligned}
$$

where $C$ is the constant as described in Theorem 2.2.

Let $T$ be a fixed time, we define the truncation errors $\tau_{1}, \tau_{2}$ as follows:

$$
\begin{aligned}
& \int_{0}^{T} \int_{0}^{l_{1}}\left(\left(1+a_{1}^{2}\right) \theta_{t}^{h}-\alpha_{1} g_{t}^{h}\right) \phi_{1} d x d t+\int_{0}^{T} \int_{0}^{l_{1}} \theta_{x}^{h} \phi_{1_{x}} d x d t=\int_{0}^{T}\left(\theta_{x}^{h} \phi_{1}\right)\left(l_{1}, t\right) d t+\tau_{1} \\
& \int_{0}^{T} \int_{l_{2}}^{1}\left(\left(d+a_{2}^{2}\right) \psi_{t}^{h}-\alpha_{2} g_{t}^{h}\right) \phi_{2} d x d t+\int_{0}^{T} \int_{l_{2}}^{1} \psi_{x}^{h} \phi_{2_{x}} d x d t=-\int_{0}^{T}\left(\psi_{x}^{h} \phi_{2}\right)\left(l_{2}, t\right) d t+\tau_{2},
\end{aligned}
$$

where $\phi_{1}, \phi_{2}$ are test functions to be described later. The next two theorems will give the exact measurements of $\tau_{1}, \tau_{2}$.

Theorem 3.2. $\quad$ Let $T$ be a fixed time and $\phi_{1}$ be a test function that satisfies

$$
\phi_{1}(0, t)=0, C\left(\phi_{1}\right)=\left\|\phi_{1}\right\|_{H^{1}\left(\left(0, l_{1}\right) \times(0, T)\right)}+\left\|\phi_{1}(x, t)\right\|_{L^{2}\left(0, l_{1}\right)}+\left\|\phi_{1}\left(l_{1}, t\right)\right\|_{L^{2}(0, T)}<\infty .
$$

If $a_{1}, a_{2}, \alpha_{1}, \alpha_{2}$ are small and $\Delta x, \Delta t$ are chosen so that they satisfy (2.1), (2.10), and (2.11), then

$$
\left|\tau_{1}\right| \leq C C_{0}^{1 / 2} C\left(\phi_{1}\right) \Delta x^{p}, 0<p \leq 1,
$$

where $C$ is the constant as described in Theorem 2.2.

Proof. For simplicity, we assume $T=N \Delta t$ for a positive integer $N$. By using schemes (1.13)-(1.20) and definitions of $\theta^{h}, g^{h}$, we get

$$
\begin{gathered}
\left(1+a_{1}^{2}\right) \theta_{t}^{h}-\alpha_{2} g_{t}^{h} \\
=\left(1+a_{1}^{2}\right)\left(\frac{\theta_{j}^{q+1}-\theta_{j}^{q}}{\Delta t}+\frac{\delta \theta_{j+1 / 2}^{q+1}-\delta \theta_{j+1 / 2}^{q}}{\Delta t}\left(x-x_{j}\right)\right)-\alpha_{1} \frac{g\left(\theta_{j}^{q+1}, \psi_{j}^{q+1}\right)-g\left(\theta_{j}^{q}, \psi_{j}^{q}\right)}{\Delta t} \\
=\delta^{2} \theta_{j}^{q+1}+\left(1+a_{1}^{2}\right) \frac{\delta \theta_{j+1 / 2}^{q+1}-\delta \theta_{j+1 / 2}^{q}}{\Delta t}\left(x-x_{j}\right)=\delta^{2} \theta_{j}^{q+1}+\left(1+a_{1}^{2}\right) \theta_{x t}^{h}\left(x-x_{j}\right) . \\
\theta_{x}^{h}=\delta \theta_{j+1 / 2}^{q}+\frac{\delta \theta_{j+1 / 2}^{q+1}-\delta \theta_{j+1 / 2}^{q}}{\Delta t}\left(t-t^{q}\right) .
\end{gathered}
$$

So $\tau_{1}$ can be simplified to

$$
\tau_{1}=\sum_{q=0}^{N-1} \sum_{j=0}^{M_{1}-1} \int_{t^{q}}^{t^{q+1}} \int_{x_{j}}^{x_{j+1}}\left(1+a_{1}^{2}\right) \theta_{x t}^{h}\left(x-x_{j}\right) \phi_{1} d x d t
$$




$$
\begin{aligned}
& +\sum_{q=0}^{N-1} \sum_{j=0}^{M_{1}-1} \int_{t^{q}}^{t^{q+1}} \int_{x_{j}}^{x_{j+1}} \frac{\delta \theta_{j+1 / 2}^{q+1}-\delta \theta_{j+1 / 2}^{q}}{\Delta t}\left(t-t^{q}\right) \phi_{1_{x}} d x d t \\
& +\sum_{q=0}^{N-1} \sum_{j=0}^{M_{1}-1} \int_{t^{q}}^{t^{q+1}} \int_{x_{j}}^{x_{j+1}}\left(\delta^{2} \theta_{j}^{q+1} \phi_{1}+\delta \theta_{j+1 / 2}^{q+1} \phi_{1_{x}}\right) d x d t-\int_{0}^{T} \theta_{x}^{h}\left(l_{1}, t\right) \phi_{1}\left(l_{1}, t\right) d t .
\end{aligned}
$$

The first two terms can be estimated by using (3.6) and (2.27) to get

$$
\begin{aligned}
& \mid \sum_{q=0}^{N-1} \sum_{j=0}^{M_{1}-1} \int_{t^{q}}^{t^{q+1}} \int_{x_{j}}^{x_{j+1}} \theta_{x t}^{h}\left(x-x_{j}\right) \phi_{1} d x d t \\
& \quad+\sum_{q=0}^{N-1} \sum_{j=0}^{M_{1}-1} \int_{t^{q}}^{t^{q+1}} \int_{x_{j}}^{x_{j+1}} \frac{\delta \theta_{j+1 / 2}^{q+1}-\delta \theta_{j+1 / 2}^{q}}{\Delta t}\left(t-t^{q}\right) \phi_{1_{x}} d x d t \mid \\
& \leq \Delta x\left\|\theta_{x t}^{h}\right\|_{L^{2}\left(\left(0, l_{1}\right) \times(0, T)\right)}\left\|\phi_{1}\right\|_{L^{2}\left(\left(0, l_{1}\right) \times(0, T)\right)} \\
& \quad+\Delta t\left(\sum_{q=0}^{N-1} \sum_{j=0}^{M_{1}-1}\left(\frac{\delta \theta_{j+1 / 2}^{q+1}-\delta \theta_{j+1 / 2}^{q}}{\Delta t}\right)^{2} \Delta x \Delta t\right)^{1 / 2}\left\|\phi_{1_{x}}\right\|_{L^{2}\left(\left(0, l_{1}\right) \times(0, T)\right)} \\
& \leq C C_{0}^{1 / 2} C\left(\phi_{1}\right)(\Delta x+\Delta t) .
\end{aligned}
$$

In order to estimate the last two terms of (3.12), we introduce a new function $\theta^{*}$ :

$$
\begin{aligned}
\theta^{*}= & \delta \theta_{j+1 / 2}^{q}+\frac{\delta \theta_{j+1 / 2}^{q+1}-\delta \theta_{j+1 / 2}^{q}}{\Delta t}\left(t-t^{q}\right)+\delta^{2} \theta_{j}^{q}\left(x-x_{j}\right) \\
& +\frac{\delta^{2} \theta_{j}^{q+1}-\delta^{2} \theta_{j}^{q}}{\Delta t}\left(x-x_{j}\right)\left(t-t^{q}\right),(x, t) \in\left(x_{j}, x_{j+1}\right] \\
& \times\left(t^{q}, t^{q+1}\right], 0 \leq j \leq M_{1}, 0 \leq q \leq N-1 .
\end{aligned}
$$

Then, the results of Theorems 2.2, 2.3 show that

$$
\left\|\theta^{*}\right\|_{H^{1}\left(\left(0, l_{1}\right) \times(0, T)\right)} \leq C C_{0}^{1 / 2} .
$$

We now simplify the last two terms of (3.12).

$$
\begin{aligned}
& \sum_{q=0}^{N-1} \sum_{j=0}^{M_{1}-1} \int_{t^{q}}^{t^{q+1}} \int_{x_{j}}^{x_{j+1}}\left(\delta^{2} \theta_{j}^{q+1} \phi_{1}+\delta \theta_{j+1 / 2}^{q+1} \phi_{1_{x}}\right) d x d t-\int_{0}^{T}\left(\theta_{x}^{h} \phi_{1}\right)\left(l_{1}, t\right) d t \\
& =\sum_{q=0}^{N-1} \sum_{j=0}^{M_{1}-1} \int_{t^{q}}^{t^{q+1}} \int_{x_{j}}^{x_{j+1}}\left(\theta_{x}^{*} \phi_{1}-\frac{\delta^{2} \theta_{j}^{q+1}-\delta^{2} \theta_{j}^{q}}{\Delta t}\left(t-t^{q}\right) \phi_{1}+\delta \theta_{j+1 / 2}^{q+1} \phi_{1_{x}}\right) d x d t \\
& =\sum_{q=0}^{N-1} \sum_{j=0}^{M_{1}-1} \int_{t^{q}}^{t^{q+1}} \int_{x_{j}}^{x_{j+1}}\left(-\theta^{*} \phi_{1_{x}}+\delta \theta_{j+1 / 2}^{q+1} \phi_{1_{x}}\right) d x d t
\end{aligned}
$$


$-\sum_{q=0}^{N-1} \sum_{j=0}^{M_{1}-1} \int_{t^{q}}^{t^{q+1}} \int_{x_{j}}^{x_{j+1}} \frac{\delta^{2} \theta_{j}^{q+1}-\delta^{2} \theta_{j}^{q}}{\Delta t}\left(t-t^{q}\right) \phi_{1} d x d t+\int_{0}^{T}\left(\theta^{*} \phi_{1}-\theta_{x}^{h} \phi_{1}\right)\left(l_{1}, t\right) d t$.

We estimate the middle term of (3.16) by using (2.11), (2.27), (3.6), and (3.14) to get

$$
\begin{aligned}
& \left|\sum_{q=0}^{N-1} \sum_{j=0}^{M_{1}-1} \int_{t^{q}}^{t^{q+1}} \int_{x_{j}}^{x_{j+1}} \frac{\delta^{2} \theta_{j}^{q+1}-\delta^{2} \theta_{j}^{q}}{\Delta t}\left(t-t^{q}\right) \phi_{1} d x d t\right| \\
& \quad \leq C \frac{\Delta t}{\Delta x}\left\|\phi_{1}\right\|_{L^{2}\left(\left(0, l_{1}\right) \times(0, T)\right)}\left(\sum_{q=0}^{N-1} \sum_{j=0}^{M_{1}-1}\left(\frac{\delta \theta_{j}^{q+1}-\delta \theta_{j}^{q}}{\Delta t}\right)^{2} \Delta x \Delta t\right)^{1 / 2} \\
& \quad \leq C C_{0}^{1 / 2} C\left(\phi_{1}\right) \Delta x^{p} .
\end{aligned}
$$

By the definitions of $\theta^{*}, \theta^{h}$, the last term of (3.16) vanishes. We finally estimate the first term of (3.16) by using the Cauchy-Schwarz inequality, (2.27) and (3.14).

$$
\begin{gathered}
\left|\sum_{q=0}^{N-1} \sum_{j=0}^{M_{1}-1} \int_{t^{q}}^{t^{q+1}} \int_{x_{j}}^{x_{j+1}}\left(-\theta^{*} \phi_{1_{x}}+\delta \theta_{j+1 / 2}^{q+1} \phi_{1_{x}}\right) d x d t\right| \\
=\mid \sum_{q=0}^{N-1} \sum_{j=0}^{M_{1}-1} \int_{t^{q}}^{t^{q+1}} \int_{x_{j}}^{x_{j+1}}\left(\frac{\delta \theta_{j+1 / 2}^{q+1}-\delta \theta_{j+1 / 2}^{q}}{\Delta t}\left(t-t^{q}\right)+\delta^{2} \theta_{j}^{q}\left(x-x_{j}\right)\right. \\
\left.+\frac{\delta^{2} \theta_{j}^{q+1}-\delta^{2} \theta_{j}^{q}}{\Delta t}\left(t-t^{q}\right)\left(x-x_{j}\right)\right) \phi_{1_{x}} d x d t \mid \\
\left.\left.\leq C\left(\phi_{1}\right)\left(\sum_{q=0}^{N-1} \sum_{j=0}^{M_{1}-1}\left(\frac{\delta \theta_{j+1 / 2}^{q+1}-\delta \theta_{j+1 / 2}^{q}}{\Delta t}\right)^{2} \Delta x \Delta t\right)^{1 / 2}\right)^{1 / 2}\right) \leq C C_{0}^{1 / 2} C\left(\phi_{1}\right) \Delta x .
\end{gathered}
$$

(3.10) follows from (3.12), (3.13), (3.16), (3.17), and (3.18).

Theorem 3.3. Let $\phi_{2}$ be a test function that satisfies

$$
\phi_{2}(1, t)=0, C\left(\phi_{2}\right)=\left\|\phi_{2}\right\|_{H^{1}\left(\left(l_{2}, 1\right) \times(0, T)\right)}+\left\|\phi_{2}(x, t)\right\|_{L^{2}\left(l_{2}, 1\right)}+\left\|\phi_{2}\left(l_{2}, t\right)\right\|_{L^{2}(0, T)}<\infty .
$$

If $a_{1}, a_{2}, \alpha_{1}, \alpha_{2}$ are small and $\Delta x, \Delta t$ are chosen to satisfy (2.1), (2.10), and (2.11), then

$$
\left|\tau_{2}\right| \leq C C_{0}^{1 / 2} C\left(\phi_{2}\right) \Delta x^{p}, 0<p \leq 1,
$$

$C$ is the constant as described in Theorem 2.2.

Proof. The proof is similar to the one for Theorem 3.2, and so is omitted. 


\section{CONVERGENCE AND ERROR BOUND}

Theorem 4.1. Let $\left\{\theta^{h}\right\},\left\{\psi^{h}\right\}$ be the approximate solutions constructed in (3.1), (3.2) and T be a positive time. If $\Delta x, \Delta t, a_{1}, a_{2}, \alpha_{1}, \alpha_{2}$ satisfy (2.1), (2.10), and (2.11), then there exists a subsequence $\left\{h_{1}\right\}$ of $\{h\}$, functions $\theta$ defined on $\left[0, l_{1}\right] \times[0, T]$, and $\psi$ defined $\left[l_{2}, 1\right] \times[0, T]$, such that

$$
\begin{gathered}
\lim _{h_{l} \rightarrow 0} \theta^{h_{l}}=\theta \text { pointwise uniformly on }\left[0, l_{1}\right] \times[0, T], \\
\lim _{h_{l} \rightarrow 0} \psi^{h_{l}}=\psi \text { pointwise uniformly on }\left[l_{2}, 1\right] \times[0, T], \\
\lim _{h_{l} \rightarrow 0} g^{h_{l}}=g(\theta, \psi) \text { pointwise uniformly on }[0, T] .
\end{gathered}
$$

Remark 4.1. $\theta, \psi$ are unique, the uniqueness will be proved in Theorem 4.3.

Proof. We can use (3.6) to show that the functions $\left\{\theta^{h}\right\},\left\{\psi^{h}\right\}$ are uniformly bounded and uniformly equicontinuous on the indicated sets. Appropriate subsequences, therefore, converge pointwise uniformly, which proves (4.1), (4.2). We now prove (4.3). Let $t \in\left(t^{q}, t^{q+1}\right]$ for some $q$, then

$$
\begin{aligned}
\left|g^{h_{l}}(t)-g\left(\theta^{h_{l}}, \psi^{h_{l}}\right)(t)\right| \leq\left|g^{h_{l}}(t)-g^{h_{l}}\left(t^{q}\right)\right| \\
\quad+\left|g^{h_{l}}\left(t^{q}\right)-g\left(\theta^{h_{l}}, \psi^{h_{l}}\right)\left(t^{q}\right)\right|+\left|g\left(\theta^{h_{l}}, \psi^{h_{l}}\right)\left(t^{q}\right)-g\left(\theta^{h_{l}}, \psi^{h_{l}}\right)(t)\right| .
\end{aligned}
$$

To estimate the first term of (4.4), we use (3.3) and an estimate similar to (2.19) to get

$$
\begin{aligned}
& \left|g\left(\theta^{h_{l}}, \psi^{h_{l}}\right)\left(t^{q}\right)-g\left(\theta^{h_{l}}, \psi^{h_{l}}\right)(t)\right| \\
& =\left|\frac{g\left(\theta^{q+1}, \psi^{q+1}\right)-g\left(\theta^{q}, \psi^{q}\right)}{\Delta t}\left(t-t^{q}\right)\right| \\
& \leq C \Delta t\left(\left(\sum_{j=0}^{M_{1}}\left(\frac{\theta_{j}^{q+1}-\theta_{j}^{q}}{\Delta t}\right)^{2} \Delta x\right)^{1 / 2}+\left(\sum_{j=M_{2}}^{M}\left(\frac{\psi_{j}^{q+1}-\psi_{j}^{q}}{\Delta t}\right)^{2} \Delta x\right)^{1 / 2}\right) \\
& \leq C \Delta t^{1 / 2}\left(\left(\sum_{q=0}^{N-1} \sum_{j=0}^{M_{1}}\left(\frac{\theta_{j}^{q+1}-\theta_{j}^{q}}{\Delta t}\right)^{2} \Delta x \Delta t\right)^{1 / 2}\right) \\
& \left.\quad+\left(\sum_{q=0}^{N-1} \sum_{j=M_{2}}^{M}\left(\frac{\psi_{j}^{q+1}-\psi_{j}^{q}}{\Delta t}\right)^{2} \Delta x \Delta t\right)^{1 / 2}\right) \\
& \leq C C_{0}^{1 / 2} \Delta t^{1 / 2} \leq C C_{0}^{1 / 2} \Delta x^{\frac{1+p}{2}} .
\end{aligned}
$$

The other two terms can be estimated similarly, we get

$$
\left|g^{h_{l}}(t)-g\left(\theta^{h_{l}}, \psi^{h_{l}}\right)(t)\right| \leq C C_{0}^{1 / 2} \Delta x^{\frac{1+p}{2}} .
$$

Since $g\left(\theta^{h}, \psi^{h}\right)$ is continuous for $\theta^{h}, \psi^{h}$, (4.3) follows from (4.1), (4.2), and (4.6).

We can further prove by using Theorem 3.1 and Theorem 4.1 that there exist new subsequences $\left\{\theta^{h_{l}}\right\},\left\{\psi^{h_{l}}\right\}$, which converge to $\theta, \psi$ in somewhat stronger sense. In order to simplify our notations, we will still use $\left\{h_{l}\right\}$ to represent the new indexes, and here is the theorem. 
Theorem 4.2. Let $\left\{\theta^{h}\right\},\left\{\psi^{h}\right\}$ be the approximate solutions constructed in (3.1), (3.2) and $T$ be a fixed time. If $\Delta x, \Delta t, a_{1}, a_{2}, \alpha_{1}, \alpha_{2}$ satisfy (2.1), (2.10), and (2.11), then there exists a subsequence $\left\{h_{l}\right\}$ of $\{h\}$, such that

$$
\begin{gathered}
\lim _{h_{l} \rightarrow 0} \theta^{h_{l}}(x, t)=\theta(x, t) \text { weakly in } L^{2}\left(\left(0, l_{1}\right) \times(0, T)\right), \\
\lim _{h_{l} \rightarrow 0} \theta_{x}^{h_{l}}(x, t)=\theta_{x}(x, t) \text { weakly in } L^{2}\left(\left(0, l_{1}\right) \times(0, T)\right), \\
\lim _{h_{l} \rightarrow 0} \theta_{t}^{h_{l}}(x, t)=\theta_{t}(x, t) \text { weakly in } L^{2}\left(\left(0, l_{1}\right) \times(0, T)\right), \\
\lim _{h_{l} \rightarrow 0} \theta^{h_{l}}\left(l_{1}, t\right)=\theta\left(l_{1}, t\right) \text { weakly in } L^{2}(0, T), \\
\lim _{h_{l} \rightarrow 0} \theta_{x}^{h_{l}}\left(l_{1}, t\right)=\theta_{x}\left(l_{1}, t\right) \text { weakly in } L^{2}(0, T), \\
\lim _{h_{l} \rightarrow 0} \psi^{h_{l}}(x, t)=\psi(x, t) \text { weakly in } L^{2}\left(\left(l_{2}, 1\right) \times(0, T)\right), \\
\lim _{h_{l} \rightarrow 0} \psi_{x}^{h_{l}}(x, t)=\psi_{x}(x, t) \text { weakly in } L^{2}\left(\left(l_{2}, 1\right) \times(0, T)\right), \\
\lim _{h_{l} \rightarrow 0} \psi_{t}^{h_{l}}(x, t)=\psi_{t}(x, t) \text { weakly in } L^{2}\left(\left(l_{2}, 1\right) \times(0, T)\right), \\
\lim _{h_{l} \rightarrow 0} \psi^{h_{l}}\left(l_{2}, t\right)=\psi\left(l_{2}, t\right) \text { weakly in } L^{2}(0, T), \\
\lim _{h_{l} \rightarrow 0} \psi_{x}^{h_{l}}\left(l_{2}, t\right)=\psi_{x}\left(l_{2}, t\right) \text { weakly in } L^{2}(0, T), \\
\lim _{h_{l} \rightarrow 0} g_{l}^{h_{l}}(t)=g_{t}(\theta, \psi)(t) \text { weakly in } L^{2}(0, T) .
\end{gathered}
$$

In addition, the functions $\theta, \varphi$ inherit the following properties:

$$
\begin{aligned}
\|\theta(x, t)\|_{H^{1}\left(\left(0, l_{1}\right) \times(0, T)\right)}+ & \left\|\theta\left(l_{1}, t\right)\right\|_{H^{1}(0, T)}+\left\|g_{t}(t)\right\|_{L^{2}(0, T)} \\
& +\|\psi(x, t)\|_{H^{1}\left(\left(l_{2}, 1\right) \times(0, T)\right)}+\left\|\psi\left(l_{2}, t\right)\right\|_{H^{1}(0, T)} \leq C C_{0}^{1 / 2} .
\end{aligned}
$$

Proof. We will just prove (4.8) in detail; (4.7), (4.9)-(4.17) can be proved similarly. (4.1) implies that

$$
\lim _{h_{l} \rightarrow 0} \theta^{h_{l}}(x, t)=\theta(x, t) \text { in distribution sense on }\left(0, l_{1}\right) \times(0, T),
$$

and so

$$
\lim _{h_{l} \rightarrow 0} \theta_{x}^{h_{l}}(x, t)=\theta_{x}(x, t) \text { in distribution sense on }\left(0, l_{1}\right) \times(0, T) .
$$

On the other hand, (3.6) proves that there is a new subsequence of $\left\{\theta_{x}^{h_{l}}\right\}$, which converges weakly on $L^{2}\left(\left(0, l_{1}\right) \times(0, T)\right)$. To simplify our notations, we still use $\left\{\theta^{h_{l}}\right\}$ to represent the new subsequence, so we have

$$
\lim _{h_{l} \rightarrow 0} \theta_{x}^{h_{l}}(x, t)=\bar{\theta}(x, t) \text { weakly on } L^{2}\left(\left(0, l_{1}\right) \times(0, T)\right)
$$


for some function $\bar{\theta}(x, t) \in L^{2}\left(\left(0, l_{1}\right) \times(0, T)\right)$. In particular,

$$
\lim _{h_{l} \rightarrow 0} \theta_{x}^{h_{l}}(x, t)=\bar{\theta}(x, t) \text { in distribution sense on }\left(0, l_{1}\right) \times(0, T) .
$$

By uniqueness, $\theta_{x}=\bar{\theta}$, which proves (4.8). (4.18) is a direct result of Theorem 3.1 and (4.7)-(4.17).

In the next theorem, we will prove that $\theta, \psi$ are solutions of (1.1)-(1.8). In fact, they are solutions in a sense that is stronger than the sense of distribution, because the test functions $\phi_{1}, \phi_{2}$ are in much weaker spaces than $C_{0}^{\infty}$.

Theorem 4.3. If $\phi_{1}$ satisfies (3.9) and $\phi_{2}$ satisfies (3.19), then the limit functions $\theta, \psi$ obtained in Theorem 4.1 are the solutions of (1.1)-(1.8). In other words, they satisfy

$$
\begin{aligned}
& \int_{0}^{T} \int_{0}^{l_{1}}\left(\left(1+a_{1}^{2}\right) \theta_{t}-\alpha_{1} g_{t}(\theta, \psi)\right) \phi_{1} d x d t+\int_{0}^{T} \int_{0}^{l_{1}} \theta_{x} \phi_{1_{x}} d x d t=\int_{0}^{T}\left(\theta_{x} \phi_{1}\right)\left(l_{1}, t\right) d t \\
& \int_{0}^{T} \int_{l_{2}}^{1}\left(\left(d+a_{2}^{2}\right) \psi_{t}-\alpha_{2} g_{t}(\theta, \psi)\right) \phi_{2} d x d t+\int_{0}^{T} \int_{l_{2}}^{1} \psi_{x} \phi_{2_{x}} d x d t=-\int_{0}^{T}\left(\psi_{x} \phi_{2}\right)\left(l_{2}, t\right) d t
\end{aligned}
$$

and

$$
\begin{aligned}
& \theta(0, t)=0,-k_{1} \theta_{x}\left(l_{1}, t\right)=k\left(\theta\left(l_{1}, t\right)-\psi\left(l_{2}, t\right)\right), \\
& \psi(1, t)=0,-k_{2} \psi_{x}\left(l_{2}, t\right)=k\left(\theta\left(l_{1}, t\right)-\psi\left(l_{2}, t\right)\right) .
\end{aligned}
$$

Proof. In (3.7), we let $\{h\}$ be the subsequence $\left\{h_{l}\right\}$ in Theorem 4.2 , and let $h_{l} \rightarrow 0$. Then (4.23) is proved by (3.9), (3.10), (4.8), (4.9), (4.10), and (4.17). (4.24) can be proved similarly. By (3.4), (4.1), (4.2), we have

$$
\lim _{h_{l} \rightarrow 0}-k\left(\theta^{h_{l}}\left(l_{1}, t\right)-\psi^{h_{l}}\left(l_{2}, t\right)\right)=-k\left(\theta\left(l_{1}, t\right)-\psi\left(l_{2}, t\right)\right) \text {, pointwise uniformly on }[0, T] .
$$

By a similar proof as (4.1), we can prove that

$$
\lim _{h_{l} \rightarrow 0} \theta_{x}^{h_{l}}=\theta_{1}(x, t) \text { pointwise uniformly on }\left[0, l_{1}\right] \times[0, T]
$$

for some function $\theta_{1}(x, t)$ defined on $\left[0, l_{1}\right] \times[0, T]$. Further, we can prove by using (4.18) that

$$
\lim _{h_{l} \rightarrow 0} \theta_{x}^{h_{l}}=\theta_{1}(x, t) \text { weakly on } L^{2}\left(\left(0, l_{1}\right) \times(0, T)\right) .
$$

By comparing (4.8) and (4.29), we conclude that $\theta_{1}=\theta_{x}$, therefore,

$$
\lim _{h_{l} \rightarrow 0} \theta_{x}^{h_{l}}(x, t)=\theta_{x}(x, t) \text { pointwise uniformly on }\left[0, l_{1}\right] \times[0, T]
$$

in particular

$$
\lim _{h_{l} \rightarrow 0} \theta_{x}^{h_{l}}\left(l_{1}, t\right)=\theta_{x}\left(l_{1}, t\right) \text { pointwise uniformly on }[0, T] .
$$

So the second part of (4.25) is obtained from (3.4), (4.27), and (4.31). (4.26) can be proved similarly.

In the next theorem, we will give the error bound estimate. 
Theorem 4.4. Let $T$ be fixed, $d, a_{1}, a_{2}, \alpha_{1}, \alpha_{2}, l_{1}, l_{2}, k_{1}, k_{2}, k$ be the physical constants described in Section I and $C$ is the constant described in Theorem 2.2. Let $a_{1}, a_{2}, \alpha_{1}, \alpha_{2}, \Delta x, \Delta t$ be chosen to satisfy (2.1), (2.10), and (2.11). Let $\left\{\theta^{h}, \psi^{h}\right\}$ be the approximate solutions constructed in (3.1), (3.2). Then there exist unique functions $\theta, \psi$ obtained in Theorem 4.1, such that they are the exact solutions of (1.1)-(1.8), and the whole sequences $\left\{\theta^{h}\right\},\left\{\psi^{h}\right\}$ converge to $\theta, \phi$, respectively, as $h \rightarrow 0$. Moreover, if for any $0 \leq t \leq T$, we define

$\eta(t)=\left[\left\|\left(\theta-\theta^{h}\right)(x, t)\right\|_{L^{2}\left(0, l_{1}\right)}+\left\|\left(\psi-\psi^{h}\right)(x, t)\right\|_{L^{2}\left(l_{2}, 1\right)}\right]+\left(\int_{0}^{t}\left(\theta\left(l_{1}, s\right)-\psi\left(l_{2}, s\right)\right) d s\right)^{2}$,

then

$$
\eta(t) \leq C C_{0}^{1 / 2}\left(\eta(0)+\Delta x^{\frac{p}{2}}\right), 0<p \leq 1 .
$$

Remark 4.2. Notice that we actually prove that the whole sequences $\left\{\theta^{h}\right\},\left\{\psi^{h}\right\}$ converge to the unique solutions $\theta, \psi$, not just converging subsequences.

Proof. We subtract (4.23) from (3.7), multiply both sides by $k_{1}$, introduce a new notation $\Delta \theta=\theta-\theta^{h}$, take $\phi_{1}=\int_{t}^{T} \Delta \theta(x, s) d s$, which satisfies (3.9), and we get

$$
\begin{aligned}
k_{1}(1+ & \left.a_{1}^{2}\right) \int_{0}^{T} \Delta \theta_{t}\left(\int_{t}^{T} \Delta \theta d s\right) d x d t+k_{1} \int_{0}^{T} \int_{0}^{l_{1}} \Delta \theta_{x}\left(\int_{t}^{T} \Delta \theta_{x} d s\right) d x d t \\
= & \alpha_{1} k_{1} \int_{0}^{T} \int_{0}^{l_{1}}\left(g_{t}(\theta, \psi)-g_{t}^{h}\right)\left(\int_{t}^{T} \Delta \theta d s\right) d x d t \\
& +k_{1} \int_{0}^{T} \Delta \theta_{x}\left(l_{1}, t\right)\left(\int_{t}^{T} \Delta \theta\left(l_{1}, s\right) d s\right) d x+k_{1} \tau_{1} .
\end{aligned}
$$

Similarly, we subtract (4.24) from (3.8), multiply both sides by $k_{2}$, introduce $\Delta \psi=\psi-\psi^{h}$, take $\phi_{2}=\int_{t}^{T} \Delta \psi(x, s) d s$, which satisfies (3.19), and combine with (4.34) to get

$$
I_{1}+I_{2}=I_{3}+I_{4}+k_{1} \tau_{1}+k_{2} \tau_{2},
$$

where

$$
\begin{gathered}
I_{1}=k_{1}\left(1+a_{1}^{2}\right) \int_{0}^{T} \int_{0}^{l_{1}} \Delta \theta_{t}\left(\int_{t}^{T} \Delta \theta d s\right) d x d t \\
\quad+k_{2}\left(d+a_{2}^{2}\right) \int_{0}^{T} \int_{l_{2}}^{1} \Delta \psi_{t}\left(\int_{t}^{T} \Delta \psi d s\right) d x d t, \\
I_{2}=k_{1} \int_{0}^{T} \int_{0}^{l_{1}} \Delta \theta_{x}\left(\int_{t}^{T} \Delta \theta_{x} d s\right) d x d t+k_{2} \int_{0}^{T} \int_{l_{2}}^{1} \Delta \psi_{x}\left(\int_{t}^{T} \Delta \psi_{x} d s\right) d x d t, \\
I_{3}=\alpha_{1} k_{1} \int_{0}^{T} \int_{0}^{l_{1}}\left(g_{t}(\theta, \psi)-g_{t}^{h}\right)\left(\int_{t}^{T} \Delta \theta d s\right) d x d t \\
\quad+\alpha_{2} k_{2} \int_{0}^{T} \int_{t_{2}}^{1}\left(g_{t}(\theta, \psi)-g_{t}^{h}\right)\left(\int_{t}^{T} \Delta \psi d s\right) d x d t,
\end{gathered}
$$


$I_{4}=k_{1} \int_{0}^{T} \Delta \theta_{x}\left(l_{1}, t\right)\left(\int_{t}^{T} \Delta \theta\left(l_{1}, s\right) d s\right) d t-k_{2} \int_{0}^{T} \Delta \psi_{x}\left(l_{2}, t\right)\left(\int_{t}^{T} \Delta \psi\left(l_{2}, s\right) d s\right) d t$.

We now estimate $I_{1}, I_{2}, I_{3}$, and $I_{4}$. The first term of $I_{1}$ can be estimated by

$$
\begin{aligned}
k_{1}\left(1+a_{1}^{2}\right) \int_{0}^{T} \int_{0}^{l_{1}} \Delta \theta_{t}\left(\int_{t}^{T} \Delta \theta d s\right) d x d t \\
=\left.k_{1}\left(1+a_{1}^{2}\right) \int_{0}^{l_{1}} \Delta \theta\left(\int_{t}^{T} \Delta \theta d s\right) d x\right|_{0} ^{T}-k_{1}\left(1+a_{1}^{2}\right) \int_{0}^{T} \int_{0}^{l_{1}}\left(-\Delta \theta^{2}\right) d x d t \\
=-k_{1}\left(1+a_{1}^{2}\right) \int_{0}^{l_{1}} \Delta \theta(x, 0)\left(\int_{0}^{T} \Delta \theta d t\right) d x+k_{1}\left(1+a_{1}^{2}\right) \int_{0}^{T} \int_{0}^{l_{1}} \Delta \theta^{2} d x d t \\
\geq-\frac{k_{1}\left(1+a_{1}^{2}\right)}{2} \int_{0}^{l_{1}} \Delta \theta(x, 0)^{2} d x-\frac{k_{1}\left(1+a_{1}^{2}\right)}{2} \int_{0}^{l_{1}}\left(\int_{0}^{T} \Delta \theta(x, t) d t\right)^{2} d x \\
\quad+k_{1}\left(1+a_{1}^{2}\right) \int_{0}^{t} \int_{0}^{l_{1}} \Delta \theta^{2} d x d t \\
\geq-\frac{k_{1}\left(1+a_{1}^{2}\right)}{2} \int_{0}^{l_{1}} \Delta \theta(x, 0)^{2} d x+k_{1}\left(1+a_{1}^{2}\right)\left(1-\frac{l_{1}}{2}\right) \int_{0}^{T} \int_{0}^{l_{1}} \Delta \theta^{2} d x d t .
\end{aligned}
$$

We can estimate the other term of $I_{1}$ similarly to get

$$
\begin{aligned}
I_{1} \geq k_{1}\left(1+a_{1}^{2}\right)(1 & \left.-\frac{l_{1}}{2}\right) \int_{0}^{T} \int_{0}^{l_{1}} \Delta \theta^{2} d x d t+k_{2}\left(d+a_{2}^{2}\right)\left(1-\frac{l_{2}}{2}\right) \int_{0}^{T} \int_{l_{2}}^{1} \Delta \psi^{2} d x d t \\
& -\frac{k_{1}\left(1+a_{1}^{2}\right)}{2} \int_{0}^{l_{1}} \Delta \theta(x, 0)^{2} d x-\frac{k_{2}\left(d+a_{2}^{2}\right)}{2} \int_{l_{2}}^{1} \Delta \psi(x, 0)^{2} d x .
\end{aligned}
$$

We now estimate the first term of $I_{2}$ to get

$$
\begin{aligned}
& k_{1} \int_{0}^{T} \int_{0}^{l_{1}} \Delta \theta_{x}\left(\int_{t}^{T} \Delta \theta_{x} d s\right) d x d t \\
& =k_{1} \int_{0}^{T} \int_{0}^{l_{1}}\left(-\frac{d}{d t} \int_{t}^{T} \Delta \theta_{x}(x, s) d s\right)\left(\int_{t}^{T} \Delta \theta_{x} d s\right) d x d t \\
& =-\left.\frac{k_{1}}{2} \int_{0}^{l_{1}}\left(\int_{t}^{T} \Delta \theta_{x}(x, s) d s\right)^{2}\right|_{0} ^{T} d x=\frac{k_{1}}{2} \int_{0}^{l_{1}}\left(\int_{0}^{T} \Delta \theta_{x}(x, t) d t\right)^{2} d x .
\end{aligned}
$$

We can estimate the other term of $I_{2}$ similarly to get

$$
I_{2}=\frac{k_{1}}{2} \int_{0}^{l_{1}}\left(\int_{0}^{T} \Delta \theta(x, t) d t\right)^{2} d x+\frac{k_{2}}{2} \int_{l_{2}}^{1}\left(\int_{0}^{T} \Delta \psi(x, t) d t\right)^{2} d x .
$$


We integrate by parts to the first term of $I_{3}$ to get

$$
\begin{aligned}
& \alpha_{1} k_{1} \int_{0}^{T} \int_{0}^{l_{1}}\left(g_{t}(\theta, \psi)-g_{t}^{h}\right)\left(\int_{t}^{T} \Delta \theta(x, s) d s\right) d x d t \\
&=\left.\alpha_{1} k_{1} \int_{0}^{l_{1}}\left(\left(g(\theta, \psi)-g^{h}\right)\left(\int_{t}^{T} \Delta \theta(x, s) d s\right)\right)\right|_{0} ^{T} d x \\
& \quad+\alpha_{1} k_{1} \int_{0}^{T} \int_{0}^{l_{1}}\left(g(\theta, \psi)-g^{h}\right) \Delta \theta d x d t \\
&=-\alpha_{1} k_{1}\left(g(\theta, \psi)-g^{h}\right)(0) \int_{0}^{T} \int_{0}^{l_{1}} \Delta \theta d x d t+\alpha_{1} k_{1} \int_{0}^{T} \int_{0}^{l_{1}}\left(g(\theta, \psi)-g^{h}\right) \Delta \theta d x d t \\
& \leq \frac{\alpha_{1} k_{1}}{2}\left(\left(g(\theta, \psi)-g^{h}\right)(0)\right)^{2}+\frac{\alpha_{1} k_{1}}{2} \int_{0}^{T} \int_{0}^{l_{1}} \Delta \theta^{2} d x d t \\
&+\frac{\alpha_{1} k_{1}}{2} \int_{0}^{T} \int_{0}^{l_{1}}\left(g(\theta, \psi)-g^{h}\right)^{2} d x d t+\frac{\alpha_{1} k_{1}}{2} \int_{0}^{T} \int_{0}^{l_{1}} \Delta \theta^{2} d x d t \\
& \leq \frac{\alpha_{1} k_{1}}{2}\left(\left(g(\theta, \psi)-g\left(\theta^{h}, \psi^{h}\right)\right)(0)\right)^{2}+\frac{\alpha_{1} k_{1}}{2}\left(\left(g\left(\theta^{h}, \psi^{h}\right)-g^{h}\right)(0)\right)^{2} \\
&+\alpha_{1} k_{1} \int_{0}^{T} \int_{0}^{l_{1}} \Delta \theta^{2} d x d t+\frac{\alpha_{1} k_{1}}{2} \int_{0}^{T} \int_{0}^{l_{1}}\left(g(\theta, \psi)-g\left(\theta^{h}, \psi^{h}\right)\right)^{2} d x d t \\
&+\frac{\alpha_{1} k_{1}}{2} \int_{0}^{T} \int_{0}^{l_{1}}\left(g\left(\theta^{h}, \psi^{h}\right)-g^{h}\right)^{2} d x d t .
\end{aligned}
$$

By using (4.5), we can estimate the second and last term of (4.44) as

$$
\frac{\alpha_{1} k_{1}}{2}\left(\left(g\left(\theta^{h}, \psi^{h}\right)-g^{h}\right)(0)\right)^{2}+\frac{\alpha_{1} k_{1}}{2} \int_{0}^{T} \int_{0}^{l_{1}}\left(g\left(\theta^{h}, \psi^{h}\right)-g^{h}\right)^{2} d x d t \leq C \Delta x^{1+p} .
$$

We now estimate the first and fourth term of (4.44). By the definition of $g$ and a known formula $\max \{r, 0\}=\frac{1}{2} r+\frac{1}{2}|r|$, we get

$$
\begin{aligned}
& g(\theta, \psi)-g\left(\theta^{h}, \psi^{h}\right) \\
& =\max \left\{a_{1} \int_{0}^{l_{1}} \theta d x+a_{2} \int_{l_{2}}^{1} \psi d x-\tilde{g} ; 0\right\}-\max \left\{a_{1} \int_{0}^{l_{1}} \theta^{h} d x+a_{2} \int_{l_{2}}^{1} \psi^{h} d x-\tilde{g} ; 0\right\} \\
& =\frac{a_{1}}{2} \int_{0}^{l_{1}} \Delta \theta d x+\frac{a_{2}}{2} \int_{l_{2}}^{1} \Delta \psi d x \\
& \quad+\frac{1}{2}\left|a_{1} \int_{0}^{l_{1}} \theta d x+a_{2} \int_{l_{2}}^{1} \psi d x-\tilde{g}\right|-\frac{1}{2}\left|a_{1} \int_{0}^{l_{1}} \theta^{h} d x+a_{2} \int_{l_{2}}^{1} \psi^{h}-\tilde{g}\right|
\end{aligned}
$$


So,

$$
\begin{aligned}
& \left|g(\theta, \psi)-g\left(\theta^{h}, \psi^{h}\right)\right| \\
& \quad \leq \frac{a_{1}}{2}\left|\int_{0}^{l_{1}} \Delta \theta d x\right|+\frac{a_{2}}{2}\left|\int_{l_{2}}^{1} \Delta \psi d x\right|+\frac{1}{2}\left|a_{1} \int_{0}^{l_{1}} \Delta \theta d x+a_{2} \int_{l_{2}}^{1} \Delta \psi d x\right| \\
& \quad \leq a_{1}\left|\int_{0}^{l_{1}} \Delta \theta d x\right|+a_{2}\left|\int_{l_{2}}^{1} \Delta \psi d x\right| .
\end{aligned}
$$

Thus,

$$
\begin{aligned}
& \frac{\alpha_{1} k_{1}}{2}\left(\left(g(\theta, \psi)-g\left(\theta^{h}, \psi^{h}\right)\right)(0)\right)^{2}+\frac{\alpha_{1} k_{1}}{2} \int_{0}^{T} \int_{0}^{l_{1}}\left(g(\theta, \psi)-g\left(\theta^{h}, \psi^{h}\right)\right)^{2} d x d t \\
& \leq C\left(\int_{0}^{l_{1}} \Delta \theta(x, 0)^{2} d x+\int_{l_{2}}^{1} \Delta \psi(x, 0)^{2} d x\right) \\
& \quad+\alpha_{1} k_{1}\left(a_{1}^{2}+a_{2}^{2}\right)\left(\int_{0}^{T} \int_{0}^{l_{1}} \Delta \theta^{2} d x d t+\int_{0}^{T} \int_{l_{2}}^{1} \Delta \psi^{2} d x d t\right) .
\end{aligned}
$$

So,

$$
\begin{aligned}
& \left|\alpha_{1} k_{1} \int_{0}^{T} \int_{0}^{l_{1}}\left(g_{t}(\theta, \psi)-g_{t}^{h}\right)\left(\int_{t}^{T} \Delta \theta(x, s) d s\right) d x d t\right| \\
& \leq C\left(\int_{0}^{l_{1}} \Delta \theta(x, 0)^{2} d x+\int_{l_{2}}^{1} \Delta \psi(x, 0)^{2} d x\right) \\
& \quad+\alpha_{1} k_{1}\left(a_{1}^{2}+a_{2}^{2}\right)\left(\int_{0}^{T} \int_{0}^{l_{1}} \Delta \theta^{2} d x d t+\int_{0}^{T} \int_{l_{2}}^{1} \Delta \psi^{2} d x d t\right) .
\end{aligned}
$$

We do a similar estimate to the other term of $I_{3}$ to get

$$
\begin{aligned}
I_{3} \leq C & \left(\int_{0}^{l_{1}} \Delta \theta(x, 0)^{2} d x+\int_{l_{2}}^{1} \Delta \psi(x, 0)^{2} d x\right) \\
& +\left(\alpha_{1} k_{1}+\alpha_{2} k_{2}\right)\left(a_{1}^{2}+a_{2}^{2}\right)\left(\int_{0}^{T} \int_{0}^{l_{1}} \Delta \theta^{2} d x d t+\alpha_{2} k_{2}\left(1+a_{2}\right) \int_{0}^{T} \int_{l_{2}}^{1} \Delta \psi^{2} d x d t\right) .
\end{aligned}
$$

Finally, we estimate $I_{4}$. By using the boundary conditions (3.2), (3.3), (4.25), and (4.26), we get

$$
\begin{aligned}
I_{4}= & -k \int_{0}^{T}\left(\Delta \theta\left(l_{1}, t\right)-\Delta \psi\left(l_{2}, t\right)\right)\left(\int_{t}^{T} \Delta \theta\left(l_{1}, s\right) d s\right) d t \\
& +k \int_{0}^{T}\left(\Delta \theta\left(l_{1}, t\right)-\Delta \psi\left(l_{2}, t\right)\right)\left(\int_{0}^{T} \Delta \psi\left(l_{2}, s\right) d s\right) d t
\end{aligned}
$$



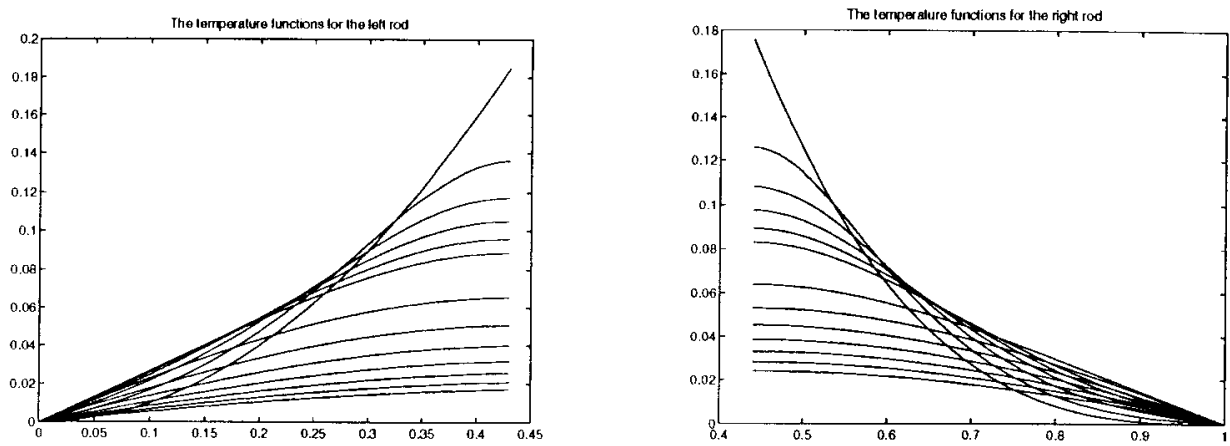

FIG. 2. Temperature functions corresponding to (5.1).

$$
\begin{aligned}
& =-k \int_{0}^{T}\left(\theta\left(l_{1}, t\right)-\psi\left(l_{2}, t\right)\right)\left(\int_{t}^{T}\left(\Delta \theta\left(l_{1}, s\right)-\Delta \psi\left(l_{2}, s\right)\right) d s\right) d t \\
& =k \int_{0}^{T}\left(\frac{d}{d t} \int_{t}^{T}\left(\Delta \theta\left(l_{1}, s\right)-\Delta \psi\left(l_{2}, s\right)\right) d s\right)\left(\int_{t}^{T}\left(\Delta \theta\left(l_{1}, s\right)-\Delta \psi\left(l_{2}, s\right)\right) d s\right) d t \\
& =\frac{k}{2}\left(\left.\int_{t}^{T}\left(\Delta \theta\left(l_{1}, s\right)-\Delta \psi\left(l_{2}, t\right) d s\right)^{2}\right|_{0} ^{T}\right. \\
& =-\frac{k}{2}\left(\int_{0}^{T}\left(\Delta \theta\left(l_{1}, t\right)-\Delta \psi\left(l_{2}, t\right)\right) d t\right)^{2} .
\end{aligned}
$$

We put the estimates of $I_{1}, I_{2}, I_{3}$, and $I_{4}$ back to (4.35) and simplify to get

$$
\begin{aligned}
& \left(k_{1}\left(1+a_{1}^{2}\right)\left(1-\frac{l_{1}}{2}\right)-\left(\alpha_{1} k_{1}+\alpha_{2} k_{2}\right)\left(a_{1}^{2}+a_{2}^{2}\right)\right) \int_{0}^{T} \int_{0}^{l_{1}} \Delta \theta^{2} d x d t \\
& \quad+\left(k_{2}\left(d+a_{2}^{2}\right)\left(1-\frac{l_{2}}{2}\right)-\left(\alpha_{1} k_{1}+\alpha_{2} k_{2}\right)\left(a_{1}^{2}+a_{2}^{2}\right)\right) \int_{0}^{T} \int_{l_{2}}^{1} \Delta \psi^{2} d x d t
\end{aligned}
$$
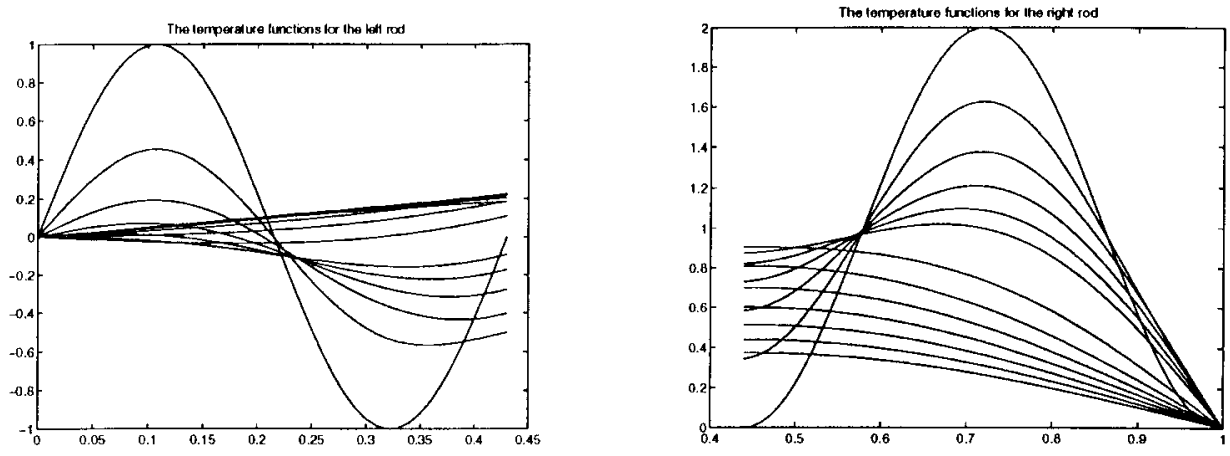

FIG. 3. Temperature functions corresponding to (5.2). 


$$
\begin{aligned}
& +\frac{k}{2}\left(\int_{0}^{T}\left(\Delta \theta\left(l_{1}, t\right)-\Delta \psi\left(l_{2}, t\right)\right) d t\right)^{2} \\
\leq & C\left(\eta^{2}(0)+C_{0} \tau_{1}+C_{0} \tau_{2}+\Delta x^{2}\right) \leq C C_{0}\left(\eta^{2}(0)+\Delta x^{p}\right) .
\end{aligned}
$$

Since $a_{1}, a_{2}, \alpha_{1}, \alpha_{2}$ are small, we can assume that

$$
\begin{aligned}
& k_{1}\left(1+a_{1}^{2}\right)\left(1-\frac{l_{1}}{2}\right)-\left(\alpha_{1} k_{1}+\alpha_{2} k_{2}\right)\left(a_{1}^{2}+a_{2}^{2}\right) \geq \frac{1}{4}, \\
& k_{2}\left(d+a_{2}^{2}\right)\left(1-\frac{l_{2}}{2}\right)-\left(\alpha_{1} k_{1}+\alpha_{2} k_{2}\right)\left(a_{1}^{2}+a_{2}^{2}\right) \geq \frac{1}{4},
\end{aligned}
$$

we then have

$$
\eta^{2}(t) \leq C C_{0}\left(\eta^{2}(0)+\Delta x^{p}\right),
$$

and (4.33) follows. Since we just proved that the whole sequences $\left\{\theta^{h}\right\},\left\{\psi^{h}\right\}$ converge, therefore $\theta, \psi$ are unique.

Remark 4.3. If in (2.9), we take $p=1$, then (2.9) will become the CFL condition, and the corresponding error bound for the case will be $\Delta x^{1 / 2}$.

\section{COMPUTATIONAL RESULTS}

In this section, we will provide some computational results to schemes (1.13)-(1.20). We take $\lambda=0.5, a_{1}=a_{2}=0.001, d=k_{1}=1, k_{2}=0.06, l_{1}=0.43, l_{2}=0.56$, and $\Delta x=0.01, \Delta t=$ $2 \Delta x^{2}$. We first take the following two initial functions:

$$
\theta(x, 0)=x^{2}, 0 \leq x \leq 0.43, \psi(x, 0)=(1-x)^{2}, 0.44 \leq x \leq 1 .
$$

Then Fig. 1 shows the two temperature functions for the left and right rod at time-steps 0, 20, $40,60,80,100,200,300,400,500,600,700$, and 800. We then take the following two initial functions:

$$
\theta(x, 0)=\sin \left(\frac{2 \pi}{0.43} x\right), 0 \leq x \leq 0.43, \psi(x, 0)=1-\cos \left(\frac{\pi}{0.56}(x-0.44)\right), 0.44 \leq x \leq 1 .
$$

Figure 2 shows the two temperature functions at the same time-steps. Both figures show that the temperatures tend to stabilize.

The author thanks Professor Endre Suli and Professor Xiao-bing Feng for many valuable discussions during her visit to IMA - University of Minnesota in June, 1997.

\section{References}

1. K. Andrews, P. Shi, M. Shillor, and S. Wright, " A parabolic system modeling the thermoelastic contacts of two rods,' Quart. Appl. Math. LIII, 53-69 (1995).

2. C. Cheng and M. Shillor, "Numerical solutions to the problem of thermoelastic contact of two rods,', Math. Comput. Modeling 17, 53-71 (1993).

3. P. Shi, M. Shillor, and X. L. Zou, " Numerical solutions to one-dimensional problems of thermoelastic contact,', Comput. Math. Appl. 22, 65-78 (1991). 
4. X. Zou, "Existence and uniqueness of a solution to a singular elastic contact problem,' Applicable Anal. 51, 139 (1993).

5. R. Zarnowski and D. Hoff, "A finite-difference scheme for the Navier-Stokes equations of onedimensional, isentropic, compressible flow,' SIAM J. Numer. Anal. 28, 78-112 (1991).

6. J. Zhao and D. Hoff, " A convergent finite-difference scheme for the Navier-Stokes equations of onedimensional, nonisentropic, compressible flow,' SIAM J. Numer. Anal. 31, 1289-1311 (1994).

7. J. Zhao, "Error bound analysis of finite-difference approximations for a class of nonlinear parabolic system in 2-space dimensions," Applic. Anal. 56, 95-108 (1995).

8. J. Zhao, "A finite difference scheme for a class of nonlinear parabolic systems in 3-D space variables,", J. Math. Anal. Applic. 203, 490-517 (1996). 